\title{
The Effect of Different Pollination on the Expression of Dangshan Su Pear MicroRNA
}

\author{
Xi Cheng, ${ }^{1}$ Chongchong Yan, ${ }^{1,2}$ Jinyun Zhang, ${ }^{1,2}$ Chenhui Ma, ${ }^{1}$ Shumei Li, ${ }^{1}$ \\ Qing Jin, ${ }^{1}$ Nan Zhang, ${ }^{1}$ Yunpeng Cao, ${ }^{1}$ Yi Lin, ${ }^{1}$ and Yongping Cai ${ }^{1}$ \\ ${ }^{1}$ School of Life Science, Anhui Agricultural University, Changjiang West Road, No. 130, Hefei 230036, China \\ ${ }^{2}$ Horticultural Institute, Anhui Academy of Agricultural Sciences, Hefei, Anhui 230031, China \\ Correspondence should be addressed to Yongping Cai; ypcaiah@163.com
}

Received 3 July 2016; Revised 18 November 2016; Accepted 11 January 2017; Published 10 April 2017

Academic Editor: Limei Qiu

Copyright (C) 2017 Xi Cheng et al. This is an open access article distributed under the Creative Commons Attribution License, which permits unrestricted use, distribution, and reproduction in any medium, provided the original work is properly cited.

\begin{abstract}
The high-throughput sequencing of pear "Dangshan Su" $\times$ "Yali" (whose fruits lignin and stone cell content are high and quality is poor) and pear "Dangshan Su" $\times$ "Wonhwang" (whose fruits with low content of lignin and stone cell and the quality are better ) found that the expressions of these two miRNAs (pyr-1809 and pyr-novel-miR-144-3p) were significantly different; their corresponding target genes encode two kinds of laccase (Pbr018935.1 and Pbr003857.1). qRT-PCR results showed that these two enzymes are involved in the formation of lignin and stone cells and the existence of these two miRNAs has a negative effect on them. It was concluded that the effect of pollination on the development of stone cells may affect the synthesis of lignin, through the regulation of laccase controlled by miRNAs, and ultimately affect the formation of stone cell and fruit quality.
\end{abstract}

\section{Introduction}

MicroRNAs (miRNAs) are small, endogenous, noncoding 20-25 nucleotide (nt) small RNAs $[1,2]$ that negatively control gene expression by cleaving or inhibiting the translation of target gene transcripts $[1,3-6]$. Since miRNAs were first reported in 1993 [7], more than 35,000 mature miRNAs have been identified from 221 species including Capra hircus, Salmo salar, and Plutella xylostella (miRBase release 21, June 2014, http://www.mirbase.org/) $[8,9]$. Plant microRNAs are released as a duplex from their primary transcripts (primiRNAs) containing stem-loop structures by RNase III enzymes. In the miRNA duplex, miRNA (guide strand) associates with argonaute (AGO) proteins to inhibit gene expression through cleavage and/or translational inhibition of target RNAs, while miRNA* (passenger strand) is often degraded [3-6]. A number of studies have suggested that miRNAs play an important role in regulating plant development [10-12], secondary metabolism, and diverse responses to stresses $[13,14]$. Kaja et al. [15] reported that mdm-miR169a, mdm-miR160e, $\mathrm{mdm}-\mathrm{miR} 167 \mathrm{~b}-\mathrm{g}$, and mdm-miR168a-b were involved in fire blight resistance in apple trees. Eldem et al. [16] validated that miRNAs (miR156, miR164, miR166, miR168, miR169, miR171, and miR395) were detected in drought responses in Prunus persica. In Populus trichocarpa [17], ptr-miR397a was identified as a negative regulator of laccase genes during the biosynthetic pathway of lignin.

Pyrus bretschneideri cv. Dangshan $\mathrm{Su}$ is a Chinese pear variety, widely grown across China and other Asian countries [18]. It is self-incompatible plants with obvious xenia phenomenon. And if undergoing self-pollination, it would not produce any fruits. Furthermore, different styles of pollination can also affect its fruit quality to a great extent [19]. In recent years, due to the deterioration of varieties and inappropriate choices of pollination tree, its fruit flavor and quality were significantly decreased. One of the most prominent manifestations is the increase in the content of stone cells, resulting in rough flesh and poor taste. It has been clarified that the stone cells are formed by the thickening of the secondary wall of the cell wall of the pulp cells and lignifications. Lignin is one of the main components of stone cells; lignin biosynthesis is closely related to the development of stone cell [20]. 
In recent years, it has been reported that miRNAs were widely involved in the regulation of pear fruit development and fruit quality, via sugar and acid metabolism and hormone signaling [21]. And no relevant report has revealed the molecular mechanism underlying different styles of pollination on pear fruits. In addition, small RNAs have been annotated for pear genome [22], and the genome wide identification of pear miRNAs was recently reported [21, 23]. However, the role of microRNA for regulatory functions is unknown in Dangshan Su pear undergoing different styles of pollination, as well as the specific microRNAs which are affected by xenia phenomenon and involved in the regulation of lignin metabolism and stone cell formation in pear fruit. Previous studies have learned "Yali" (P. bretschneideri Rehd.) as male parent, whose fruit lignin and stone cell content are high and quality is poor, while the fruits with low content of lignin and stone cell have better quality, using "Wonhwang" ( $P$. pyrifolia Nakai) as the male parent. Thus, in this study, we used "Yali" and "Wonhwang" to pollinate "Dangshan Su" and built "Dangshan Su" microRNA expression profiles under different pollen donators using Solexa high-throughput sequencing technology. With bioinformatics method, the xenia phenomenon effect on "Dangshan Su" microRNA expression levels was analyzed to predict differently expressed microRNAs and their corresponding target genes. Furthermore, the lignin metabolismrelated enzyme genes and its corresponding microRNA expression changes were determined using Poly ( $T$ ) method and real-time fluorescent quantitative PCR (qRT-PCR) assay at different developmental stages of "Dangshan Su" $\times$ "Yali" and "Dangshan Su" $\times$ "Wonhwang", respectively. The above researches can lay a foundation for the regulation of lignin and stone cell formation and improve the quality of pear fruit by exploring the related miRNA which control the lignin synthesis and stone cell development, which also provide a certain theoretical basis and technical methods for subsequent research on xenia affecting fruit quality by regulating miRNA expression in which way. These results also could provide an important theoretical basis for the screening of suitable "Dangshan Su" pollinated varieties and the improvement of "Dangshan Su" fruit quality.

\section{Materials and Methods}

2.1. Materials. Fruits were obtained from 50-year-old pear trees grown on an orchard localized at Dangshan County, Anhui, China. The "Dangshan Su" female parents were fertilized with pollens from "Yali" (P. bretschneideri Rehd.) and "Wonhwang" (P. pyrifolia Nakai) male parents. Pollens were collected from "Yali" and "Wonhwang" flower androecia and used to manually pollinate "Dangshan Su," respectively. At the end of pollination processing, flowers were covered with bags for seven days. Pear fruits of different male parent pollination were collected after pollination at 23 days after flowering and the samples were taken every 8 days (120 pear fruits were taken from $47 \mathrm{~d}, 63 \mathrm{~d}, 79 \mathrm{~d}$, and $145 \mathrm{~d}$ after flowering and 40 pears at other times) and stored at $-80^{\circ} \mathrm{C}$ in our laboratory at Anhui Agricultural University, China.
2.2. Small RNA Sequencing and Library Construction. For each group, total RNA was extracted using the pBiozol Total RNA Extraction Reagent (BioFlux) following the manufacturer's instructions. All RNA samples were examined for protein contamination (A260/A280 ratios) and reagent contamination (A260/A230 ratios) by using a Nanodrop ND 1000 spectrophotometer (NanoDrop, Wilmington, DE). Before library construction, the RNAs of the "DangshanSu" $\times$ "Yali" (DangsuA) or "DangshanSu" $\times$ "Wonhwang" (DangsuB) were pooled together to form sample groups, respectively.

For library construction, total RNA was firstly resolved on denatured $15 \%$ polyacrylamide gels and 18-30 nt gel fragments were excised. A pair of adaptors was ligated to both ends of the eluted small RNA fragments. Subsequently, the adapter-ligated small RNAs were reversely transcribed into cDNA with Super-Script II Reverse Transcriptase (Invitrogen) and amplified by PCR. Finally, the resulting library was quantified and sequenced by using the Illumina HiSeq 2000 platform (BGI, Shenzhen, China).

2.3. Sequencing Data Analysis. For the raw data, all low quality reads, such as reads with adapter contaminants or poly A sequences, were filtered. After sequencing, the sequences that are longer than $30 \mathrm{nt}$ or shorter than $18 \mathrm{nt}$ and without adapters were removed, the remaining ones were used for subsequent analysis.

The clean sequencing data were mapped into two genome and Rfam database v1l (http://www.sanger.ac.uk/Software/ $\mathrm{Rfm} /$ ). Reads aligned in the genome, excluding those matching tRNAs, rRNAs, snRNA, and snoRNAs, were used for further analysis. Mature miRNAs and their precursors were retrieved from miRBase (version 21; http://www.mirbase.org) $[24,25]$.

2.4. miRNA Identification and Quantitation. The remaining reads were applied to predicting novel miRNAs and quantitative analysis using the miRDeep 2 with the parameters for plants described in miREvo. To assess the reliability of predicted novel microRNAs, their MFEI (minimum folding free energy index) were calculated to compare with the known microRNAs [26-28].

The frequency of microRNAs from different libraries was normalized by total clean reads of microRNAs in each sample group. If the normalized read count of a given microRNA is zero, the expression value was modified to 1 for further analysis.

2.5. Identification of Target Genes for Differentially Expressed miRNAs. The expression differences between small RNA libraries of both samples were determined using EdgeR package with $\mathrm{R}$ language. Then, tools named "TAPIR" and "TargetFinder" were used for the microRNAs target prediction, which was evaluated with a better prediction performance [29]. The target RNA sequences were parsed from pear genomes with their corresponding annotation files (http:// peargenome.njau.edu.cn/) [22].

2.6. Functional Enrichment and Cluster Analysis for Differential miRNAs' Target Transcripts. In order to understand the 
function of the differentially expressed genes, hyper geometric distribution test was carried out to identify GO functions and KEGG pathways in which differentially expressed genes are significantly enriched $p$ value $<0.05$, comparing total transcripts. This method firstly maps all target gene candidates to GO terms in the database (http://www.geneontology .org/), calculating gene numbers for each term, then using hypergeometric test to find significantly enriched GO terms in target gene candidates comparing to the reference gene background. The calculating formula is

$$
P=1-\sum_{i=0}^{m-1} \frac{\left(\begin{array}{c}
M \\
i
\end{array}\right)\left(\begin{array}{c}
N-M \\
n-i
\end{array}\right)}{\left(\begin{array}{c}
N \\
n
\end{array}\right)}
$$

In the formula above, $N$ is the number of all genes with GO annotation; $n$ is the number of target gene candidates in $N$; $M$ is the number of all genes that are annotated to a certain GO term; and $m$ is the number of target gene candidates in $M$. We used the Bonferroni Correction for the $p$ value to obtain a corrected $p$ value. GO terms with corrected $p$ value $\leq 0.05$ are defined as significantly enriched in target gene candidates.

KEGG calculating formula is the same as that in GO analysis. Here $N$ is the number of all genes with KEGG annotation, $n$ is the number of target gene candidates in $N, M$ is the number of all genes annotated to a certain pathway, and $\mathrm{m}$ is the number of target gene candidates in $M$. Genes with FDR $\leq 0.05$ are considered as significantly enriched in target gene candidates. The KEGG analysis could reveal the main pathways which the target gene candidates are involved in.

And differentially expressed genes hierarchically clustered to represent the expression patterns by using ward method with Euclidean distance as a measurement of similarity. All above analyses were programmed in python 2.7.5 with numpy (1.9.2), scipy (0.15.1), and matplotlib (1.4.3).

2.7. Extraction and qRT-PCR Analysis for miRNAs. To identify significantly expressed miRNAs between "Dangshan Su" $\times$ "Yali" and "Dangshan Su" $x$ "Wonhwang", miRNA quantification from fruit samples at $15 \mathrm{~d}, 23 \mathrm{~d}, 31 \mathrm{~d}, 47 \mathrm{~d}, 55 \mathrm{~d}, 63 \mathrm{~d}$, and $145 \mathrm{~d}$ after flowering (DAF) was performed using Poly (T) RT-PCR. To produce miRNA fused with Poly (T) cDNA, $0.5 \mathrm{mg}$ total RNA was used for the reverse transcription with miRNA mature sequence specific Poly (A) RT primers according to the Poly (T) RT-PCR protocol [30]. The 5s rRNA was used as an internal reference [21]. All the primers were listed in the Supplementary Material_1 (see Supplementary Material available online at https://doi.org/10.1155/2017/ 2794040). qRT-PCR analysis on cDNA samples at different developmental stages from two pollinated pear varieties was carried out with three repeats. Relative gene expression levels were calculated by the $2^{-\Delta \Delta C T}$ method [31]. Reactions contained the following: $10 \mu \mathrm{L}$ of $2 \mathrm{x}$ TransStar ${ }^{\circledR}$ Tip Green qPCR SuperMix, $2 \mu \mathrm{L}$ of template cDNA, $0.4 \mu \mathrm{L}$ of forward and Universal miRNA qPCR Primer, $0.4 \mu \mathrm{L}$ of Passive Reference Dye $(50 \mathrm{x})$, and water to $20 \mu \mathrm{L}$. PCR amplification was carried out as follows: $50^{\circ} \mathrm{C}$ for $2 \mathrm{~min}, 94^{\circ} \mathrm{C}$ for $34 \mathrm{~s}$, followed by 40 cycles of $94^{\circ} \mathrm{C}$ for $5 \mathrm{~s}$, and $60^{\circ} \mathrm{C}$ for $30 \mathrm{~s}$.
2.8. RNA Extraction and qRT-PCR Analysis for Target Genes. To examine the expression of target genes in "Dangshan Su" $\times$ "Yali" and "Dangshan Su" $\times$ "Wonhwang," fruit samples at $15 \mathrm{~d}, 23 \mathrm{~d}, 31 \mathrm{~d}, 47 \mathrm{~d}, 55 \mathrm{~d}, 63 \mathrm{~d}$, and $145 \mathrm{~d}$ after flowering (DAF) were used. Total RNAs from samples were extracted using the Trizol reagent (Invitrogen) according to the manufacturer's instructions. The DNase-treated RNA was reverse-transcribed using M-MLV reverse transcriptase (Invitrogen).

Primers (Supplementary Material_1) for qRT-PCR were designed using Primer Express 3.0 software (Applied Biosystems), and the Tubulin gene (GenBank: AB239680.1) was used as an internal reference with primers synthesized by Sangon Biotech Co., Ltd. (Shanghai, China). qRT-PCR was carried out as above mentioned. Reaction system contained $10 \mu \mathrm{L}$ of SYBR Premix Ex Taq II (2x), $1 \mu \mathrm{L}$ of template cDNA, $0.5 \mu \mathrm{L}$ of forward and reverse primer, and water to $20 \mu \mathrm{L}$. PCR amplification was carried out using the following program: $50^{\circ} \mathrm{C}$ for $2 \mathrm{~min}, 95^{\circ} \mathrm{C}$ for $30 \mathrm{~s}$, followed by 40 cycles of $95^{\circ} \mathrm{C}$ for $15 \mathrm{~s}, 60^{\circ} \mathrm{C}$ for $20 \mathrm{~s}$, and $72^{\circ} \mathrm{C}$ for $20 \mathrm{~s}$.

2.9. Determination of Stone Cell Content. Stone cell content was measured as described by Cai et al. [32] Pulp (5 g) from between $2 \mathrm{~mm}$ beneath the peel to $0.5 \mathrm{~mm}$ from the core was collected and stored at $-20^{\circ} \mathrm{C}$ for $24 \mathrm{~h}$ then homogenized at $20,000 \mathrm{rpm}$ for $3 \mathrm{~min}$. Homogenized pulp was incubated in water; the upper suspension was decanted, and this procedure was repeated several times. Stone cells were oven dried and weighed three times, and the content was calculated as follows: (stone cell content $(\%)=$ weight of stone cells ( $\mathrm{g} D W) /$ weight of pulp $(\mathrm{g} \mathrm{FW}) \times 100)$.

2.10. Determination of Lignin Content. Lignin content was measured using the Klason method [33]. Pulp (5g) from between $2 \mathrm{~mm}$ beneath the peel and $0.5 \mathrm{~mm}$ from the core was collected, oven dried, ground into a uniform powder, and passed through a 200-mesh sieve. The powder was extracted with methanol and oven dried. A small amount $(0.2 \mathrm{~g})$ of this powder was extracted with $15 \mathrm{~mL}$ of $72 \% \mathrm{H}_{2} \mathrm{SO}_{4}$ at $30^{\circ} \mathrm{C}$ for $1 \mathrm{~h}$, combined with $115 \mathrm{~mL}$ of distilled water, and boiled for $1 \mathrm{~h}$. The volume was kept constant during boiling. The mixture was filtered and the residue was rinsed with $500 \mathrm{~mL}$ of hot water, air dried, and weighed.

\section{Results and Discussion}

3.1. RNA Library Construction, Sequencing, and Data Analysis. To characterize the difference of small RNA expression levels between "Dangshan Su" $\times$ "Yali" (DangsuA) and "Dangshan Su" $\times$ "Wonhwang" (DangsuB), small RNA libraries were constructed for DangsuA and DangsuB, respectively. Using deep sequencing, a total of 10,830,189 and 9,925,992 reads were generated from DangsuA and DangsuB, respectively. After removing adapter sequences and low-quality reads, $10,809,068(99.81 \%)$ and 9,906,978 (99.81\%) clean reads from each sample group were maintained for analysis (Table 1).

All these reads from both samples were annotated to small RNA sequences deposited in the Rfamll.0 database (http://rfam .janelia.org/). Using "blastn" program with command "blastnshort task," we separated the small RNAs that matched other 
TABLE 1: Distribution of counts of sequ-seqs type in 2 libraries.

\begin{tabular}{lcccc}
\hline Type & \multicolumn{2}{c}{ DangsuA } & & DangsuB \\
& Count & Percent (\%) & 9925992 & Percent (\%) \\
\hline Total reads & 10830189 & & 9906978 & $99.81 \%$ \\
High reads & 10809068 & $99.81 \%$ & 21055 & $0.21 \%$ \\
3' adapter null & 25767 & $0.24 \%$ & 30237 & $0.31 \%$ \\
Insert null & 38463 & $0.36 \%$ & 5888 & $0.06 \%$ \\
5' adapter contaminants & 7239 & $0.07 \%$ & 31796 & $0.32 \%$ \\
Smaller than 18 nt & 43248 & $0.40 \%$ & 1082 & $0.01 \%$ \\
PolyA & 766 & $0.01 \%$ & 9816920 & $99.09 \%$ \\
Clean reads & 10693585 & $98.93 \%$ & & \\
\hline
\end{tabular}

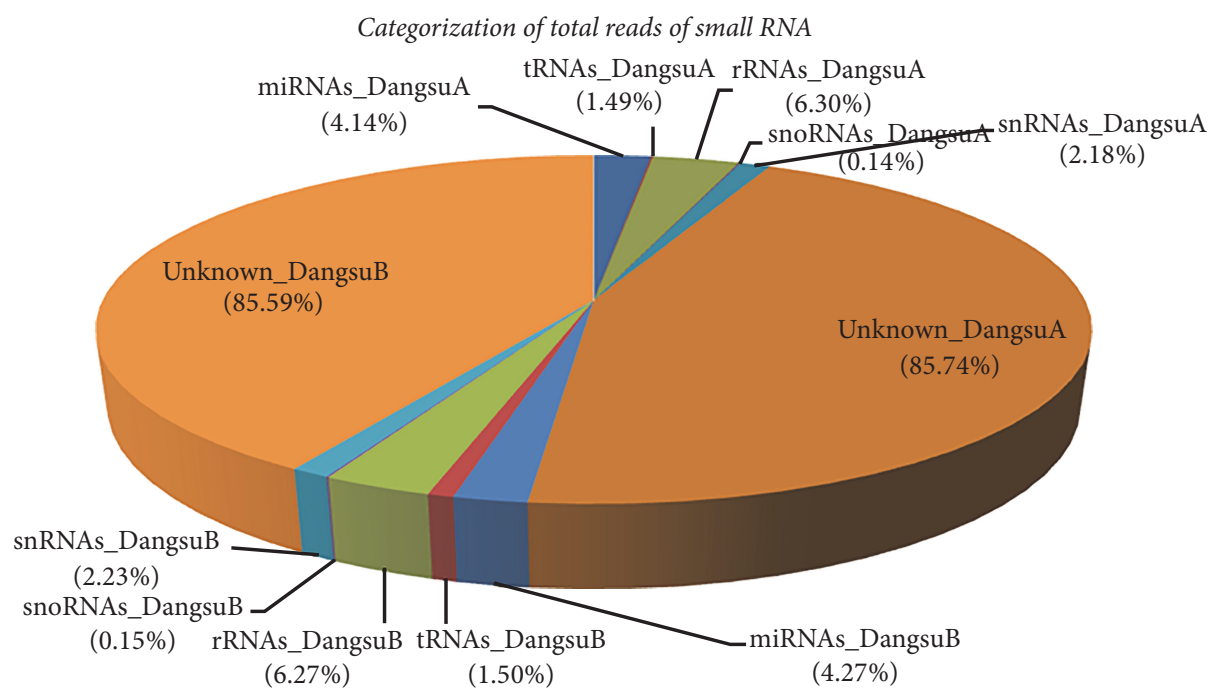

(a)

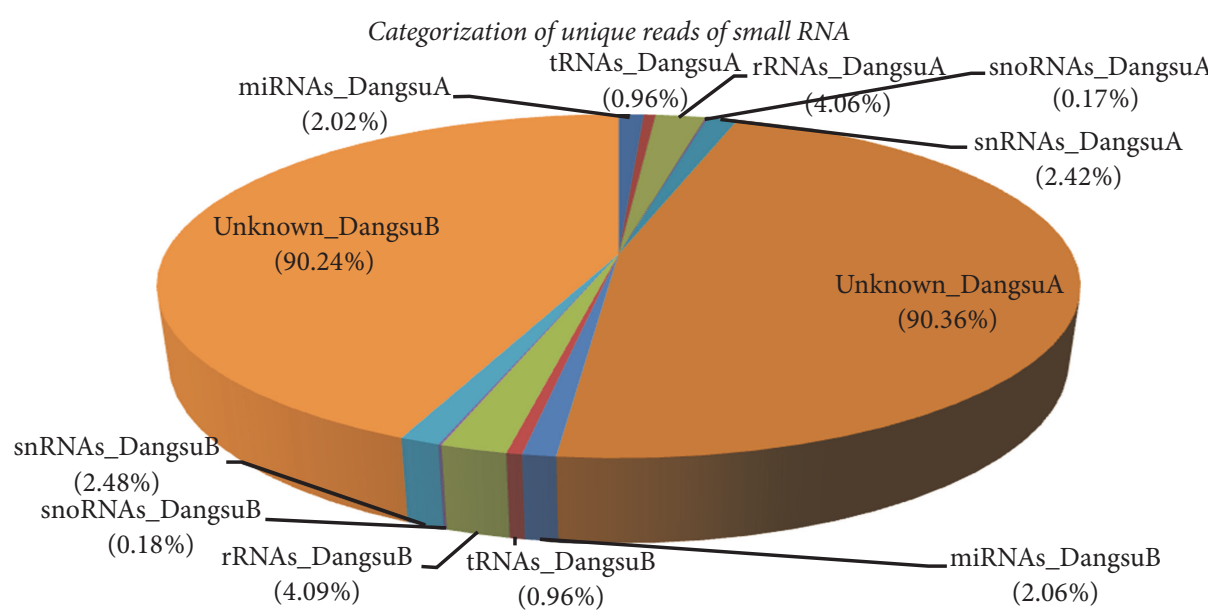

(b)

FIGURE 1: Categorization of total reads and unique reads of small RNA.

noncoding sequences, such as ribosomal RNA (rRNA), small nuclear RNA (snRNA), small nucleolar RNA (snoRNA), and transfer RNA (tRNA). The annotation of these small RNAs is listed in Figure 1. The remaining reads were considered as potential miRNAs products with various lengths of fragments
(Figure 2). The 21-24 nt sequences accounted for $93.86 \%$ and $94.01 \%$ of the total clean reads in DangsuA and DangsuB, respectively (Figure 2). Both samples shared a similar distribution pattern, in which the $24 \mathrm{nt}$ reads were the most abundant class (63.85\% in DangsuA, 66.62\% in DangsuB). 
TABLE 2: Distribution of sequence reads mapped to genome.

\begin{tabular}{lcccc}
\hline Sample name & Type & Numbers & Mapped & Unmapped \\
\hline DangsuA & Unique reads & 6074111 & $3514387(57.858 \%)$ & $2559724(42.142 \%)$ \\
DangsuB & Unique reads & 5649359 & $3275734(57.984 \%)$ & $2373625(42.016 \%)$ \\
DangsuA & Total reads & 9557093 & $5134308(53.722 \%)$ & $4422785(46.278 \%)$ \\
DangsuB & Total reads & 8773522 & $4742027(54.049 \%)$ & $4031495(45.951 \%)$ \\
\hline
\end{tabular}

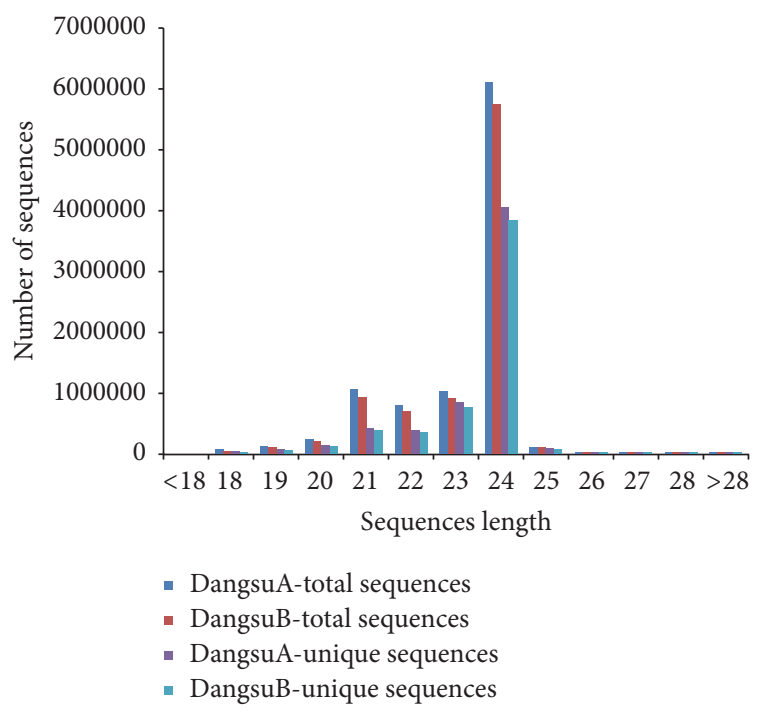

FIGURE 2: The length distribution of small RNA.

This result is consistent with other species, such as tomato [34], Citrus trifoliate [35], and Medicago truncatula [36], which is the typical size range for dicer-derived products in plants [37].

The remaining reads were then aligned to the pear genomes (http://peargenome.njau.edu.cn/), generating 53.72\% and 54.05\% genome-matched reads within DangsuA and DangsuB libraries, respectively (Table 2 and Figure 3 ). This mapping rate is lower than that in other research [21], due to the usage of alignment parameter "bowtie -n 1-1 18 -a -m 30 -best -strata." These small RNA reads were grouped into several RNA classes including rRNA, tRNA, snRNA, and snoRNA. The remaining unclassified small RNA reads contained the highest fraction of unique and total clean reads, which are likely composed of some known miRNAs and new types of regulatory novel miRNAs.

\subsection{Identification of Both the Known and Novel MicroRNAs}

3.2.1. Identification of the Known MicroRNAs. Due to the lack of pear miRNA related information within the miRBase 21 database (announced in June 2014), we had to compare our measured sRNA sequences with miRNA precursor/miRNA sequences from other published plants within the miRBase21 database, in order to display sequences and quantity of miRNA families from the samples tested, regardless of species.
After normalizing the miRNA expression levels of both DangsuA and DangsuB, 617 types of the known microRNAs were identified. Out of these microRNAs, 451 are present in both sample groups and other 71 species; 82 and 84 miRNAs are specifically expressed in DangsuA and DangsuB and distributed among 44 and 42 species, respectively (Supplementary Material_2).

The most common species, matching the microRNAs in DangsuA and DangsuB, are Malus domestica and Glycine max, each with 32 matches, followed by Prunus persica with 31, Arabidopsis thaliana with 28, and Vitis vinifera with 27 matches, respectively (Figure 4(a)). Species Oryza sativa with 9, Populus trichocarpa with 8, and Cucumis melo with 7 matches were the DangsuA specific ones (Figure 4(b)). The 11 miRNAs that were expressed in DangsuB were matched in Aegilops triaristata, while Arabidopsis, Brachypodium distachyon, and Medicago truncatula all had 7 miRNAs, respectively (Figure 4(c)). As shown in Figure 4, although apples and beans both have 32 microRNA matches with our samples, there are a total of 413 microRNAs announced for Malus domestica and 1215 for Glycine max on the whole in the miRbase database [25]. Moreover, microRNA matching rate for Malus domestica and Glycine max is $7.75 \%$ and $2.63 \%$, respectively, indicating that the species relationship should be correlated with the microRNA matching rate.

3.2.2. Identification of Novel miRNAs. Iconic hairpin structure of miRNA precursors can be used to predict new miRNA. Through the interception of a certain length of sRNA to compare with the pear genome, we can explore the secondary structure, Dicer enzyme cutting site, energy, and other characteristics information and predict new microRNA by software Mireap (http://sourceforge.net/projects/mireap/). Compared with other RNAs, MFEI of novel microRNAs larger than 0.85 are relatively more credible (Figure 5) [28]. A total of 168 and 106 new kinds of miRNAs were found in DangsuA and DangsuB, respectively (Supplementary Material_3). The mature sequence length of these novel miRNAs is within the range of 18-24 nt, with both $21 \mathrm{nt}$ and $24 \mathrm{nt}$ dominant. It can be predicted that the length of premiRNA sequences is within the range of 62 294 nt, and minimum hairpin folding free energy (MFE) has the range of $168.4 \mathrm{kcal} / \mathrm{moL} \sim$ $-20.9 \mathrm{kcal} / \mathrm{moL}$, minimum folding free energy index (MFEI) is in the range between 0.45 and 2.09 , with the majority of 0.85 . All above information indicated high reliability prediction of the novel microRNAs (Figure 6). The predictive novel miRNA precursor sequence can form a good stem-loop structure (Figure 7), which served as another evidence for high reliability prediction of the novel microRNAs. 

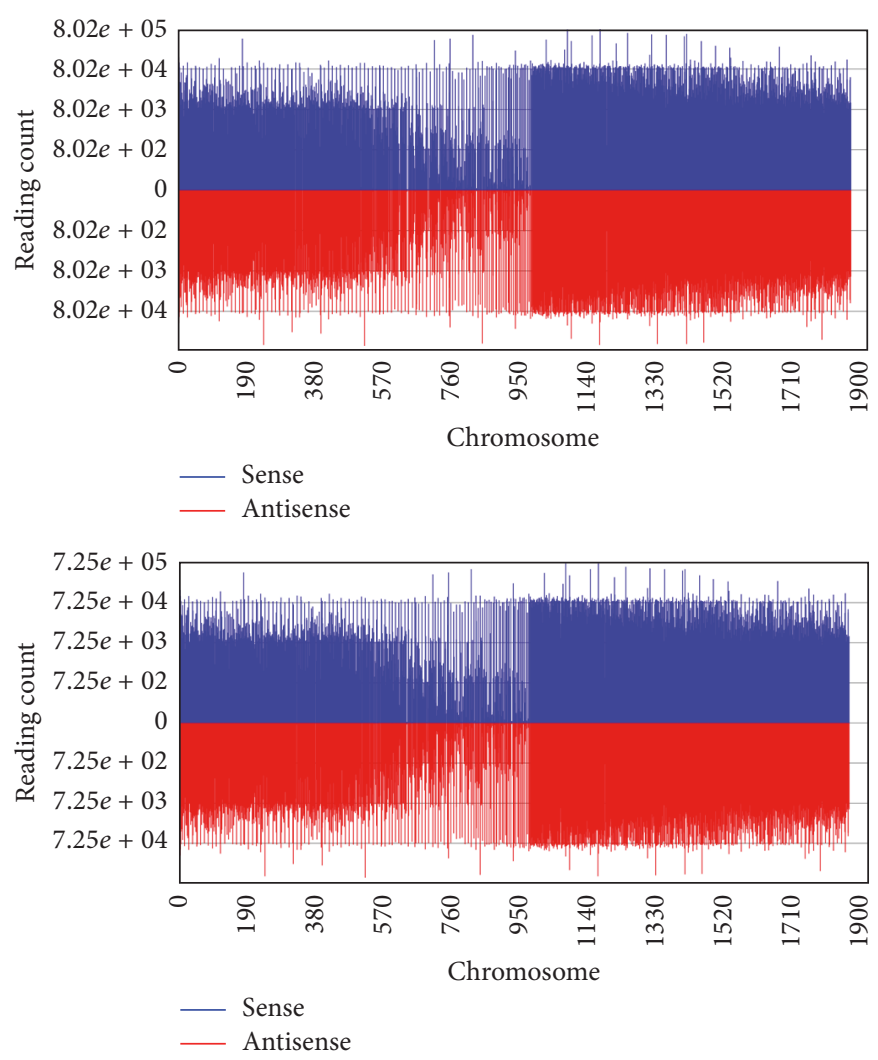

FIGURE 3: Distribution of sequence reads mapped to pear genome.

There exists a certain degree of base preference at different sites in microRNA sequences, which helps in not only microRNA cutting, but also target gene identification and other functions. For example, the U-base of the first mature sequence $\left(5^{\prime}\right)$ is characterized to help in microRNA identification by ARGONAUTEI. Conservative small molecule RNA was subjected to base preferences analysis to initially determine the reliability of the resulting sequence. The results showed that most of the first base of known and novel microRNAs were $U$, and the known microRNAs had substantially equal nucleotide contents for A, C, and G, while the novel ones had relatively more $\mathrm{C}$ nucleotide content but relatively less of the $\mathrm{G}$ base (Figure 8).

3.3. Cluster Analysis of MicroRNAs from the Differently Pollinated "Dangshan Su". Expression pattern analysis of these microRNAs indicated that the expression quantity of some microRNAs in DangsuB (Figure 9(b)) was higher than those in DangsuA (Figure 9(a)), while other microRNAs showed a reversed pattern. As showed in Figure 9(a), pyr-miR3229, pyr-miR1557, pyr-miR3925, and other known microRNAs had less expression quantity in DangsuB than in DangsuA; pyr-miR595, pyr-miR3496, and others in DangsuA and DangsuB had the same expression quantity basically; and pyrmiR870, pyr-miR518, pyr-miR753, and so forth apparently had higher expression quantity in DangsuB than in DangsuA. Data from Figure 9(b) showed that pyr-novel-miR-1243p, pyr-novel-miR-107-5p, pyr-novel-miR-160-5p, and other novel microRNAs were less expressed in DangsuB than in DangsuA; pyr-novel-miR-61-5p, pyr-novel-miR-49-3p, and so forth were consistently expressed in both; and pyrnovel-miR-65-5p, pyr-novel-miR-49-3p, pyr-novel-miR-79$3 p$, and others expression quantity was significantly higher in DangsuB than in DangsuA; and the pyr-novel-miR-150-3p, pyr-novel-miR-109-3p, and so forth were only expressed in DangsuA; and the pyr-novel-miR-159-3p, pyr-novel-miR-80$3 \mathrm{p}$, and so forth were only expressed in DangsuB.

3.4. The Variance Analysis of MicroRNA in "Dangshan Su" Different Pollination. To check out if there are significant differences in expression levels between two samples, we performed the microRNA expressed statistics in DangsuA and DangsuB using microRNA edgeR (R package). With volcano plot analysis (Figure 10), the overall distribution of differential microRNAs can be inferred, based on fold changes and the significant level ( $p$ value $<0.05$ ).

As showed in Figure 11, a total of 68 kinds of microRNAs with significant expression differences were identified in both samples (DangsuA and DangsuB). And 42 microRNAs had the higher expression quantity in DangsuB than in DangsuA, where 23 kinds are known microRNAs and 19 kinds are novel microRNAs. 26 microRNAs had less expressions in DangsuB than in DangsuA, where 16 kinds are known microRNAs and 10 kinds are novel microRNAs. In DangsuB, pyr-miR870 and pyr-novel-miR-65-5p showed $2^{6.2}$ and $2^{3.3}$ times higher than those in DangsuA. There are some microRNAs, such 


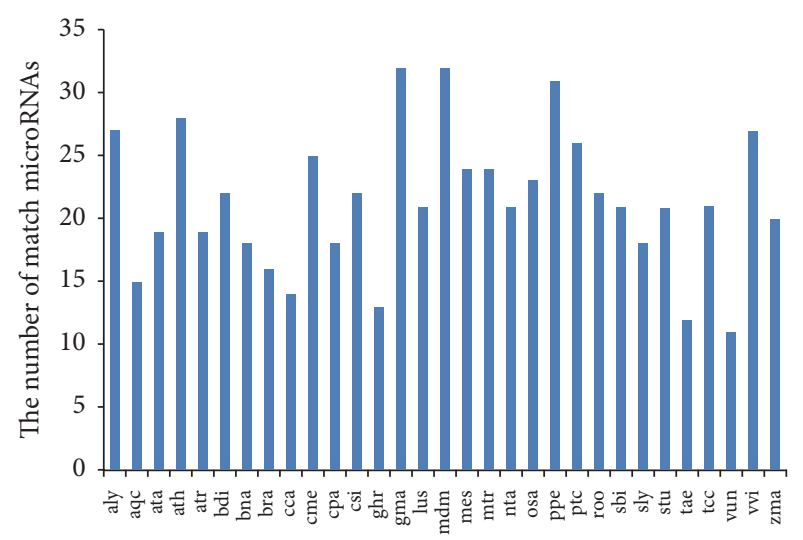

The species name

(a)

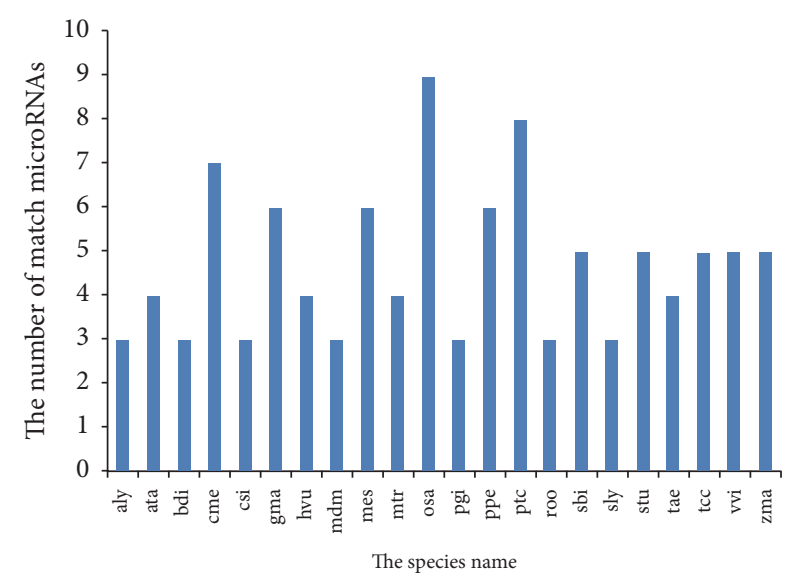

(b)

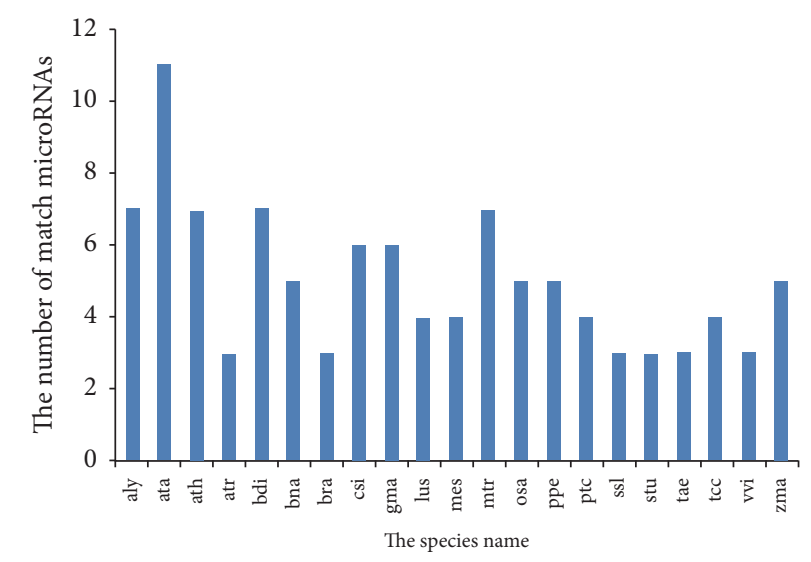

(c)

Figure 4: (a) Known miRNA matching number in different species. (b) Known miRNA matching number in DangsuA. (c) Known miRNA matching number in DangsuB.

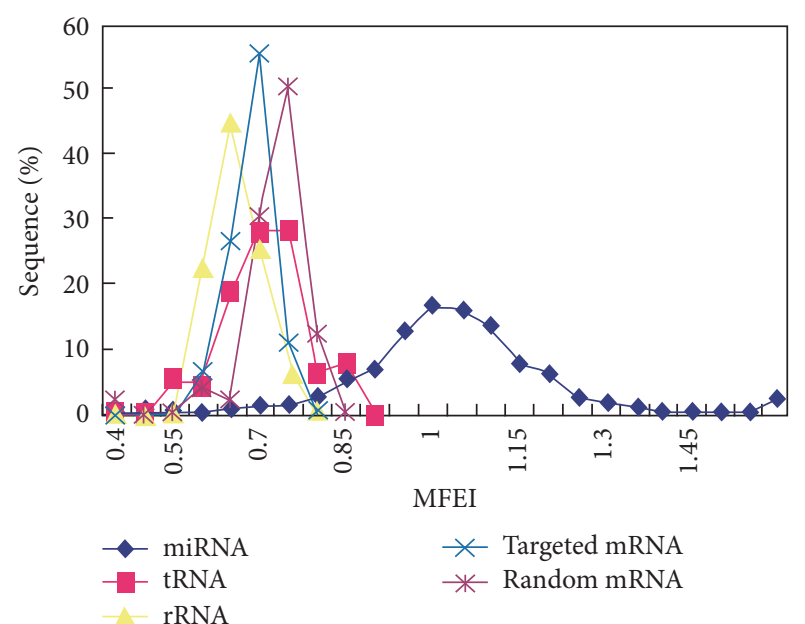

FIGURE 5: Comparison of the minimum folding free energy index (MFEI) of miRNA precursors with other RNAs.

as pyr-miR3329 and pyr-novel-miR-10-3p, with the significantly reduced expression (Supplementary Material_4). These results suggested that difference in pollination should be

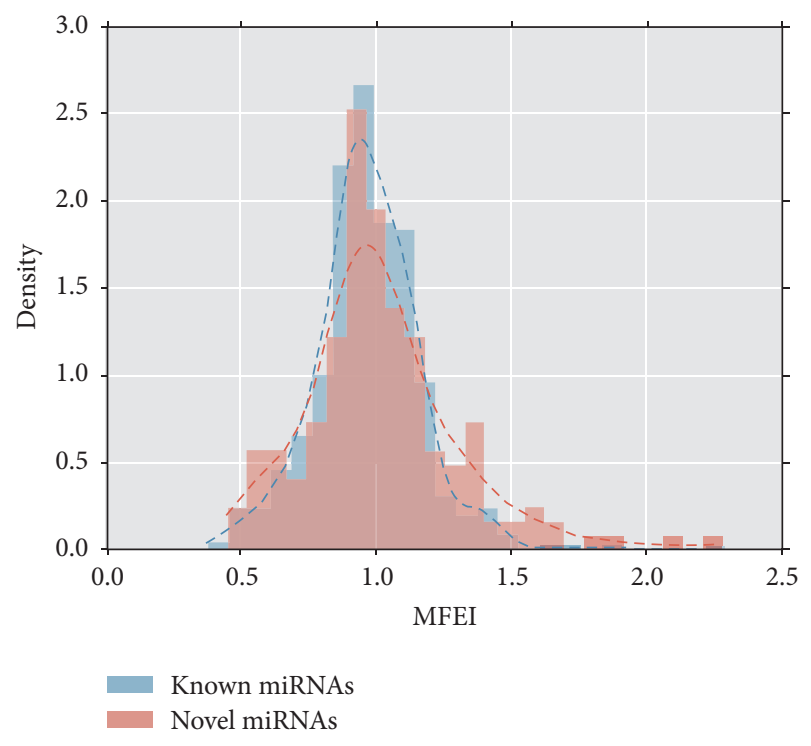

FIGURE 6: The MFEI of the candidate novel miRNA.

able to significantly alter some microRNAs expression in "Dangshan Su." 


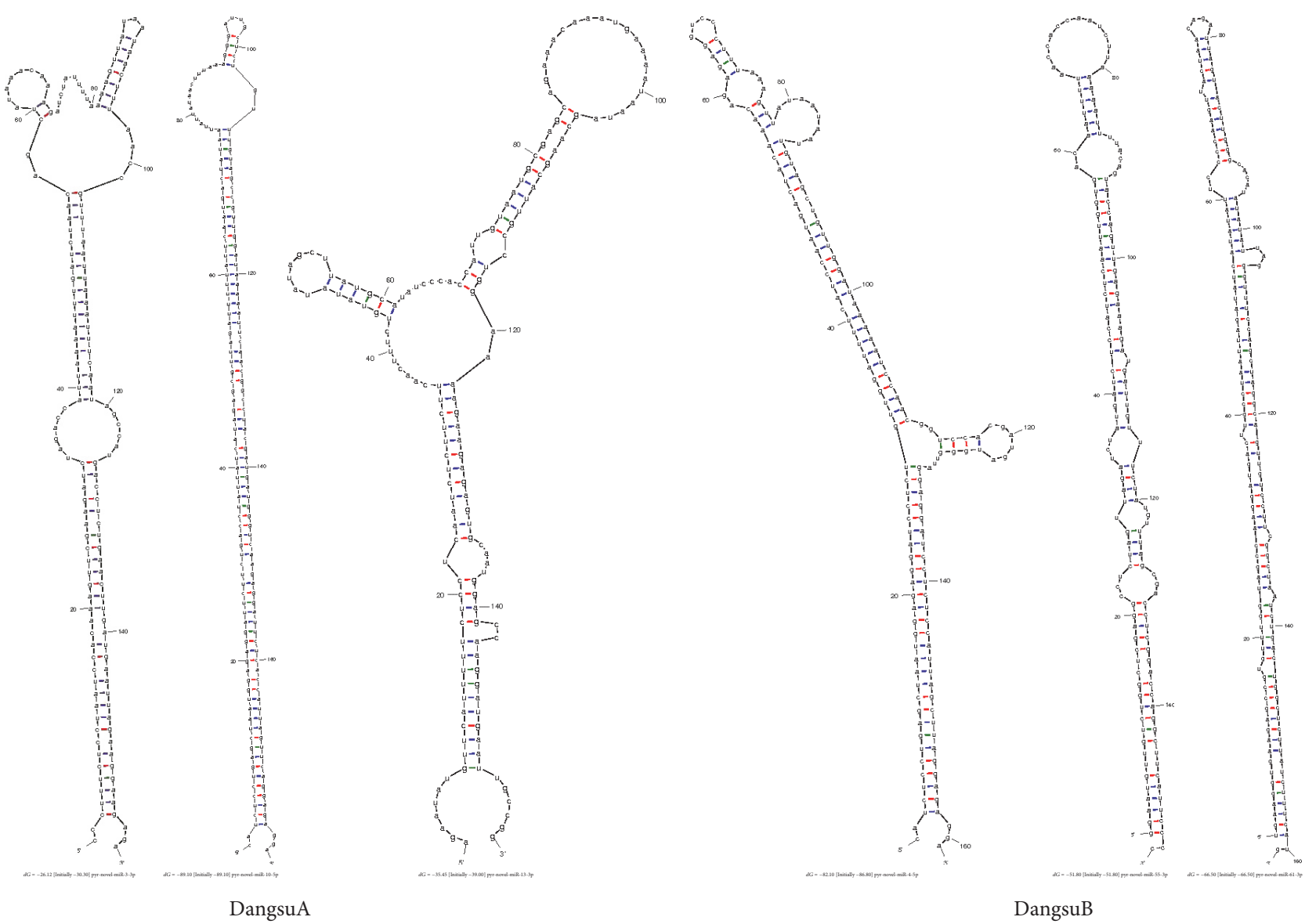

FIGURE 7: The stem-loop structure of the candidate novel miRNA.

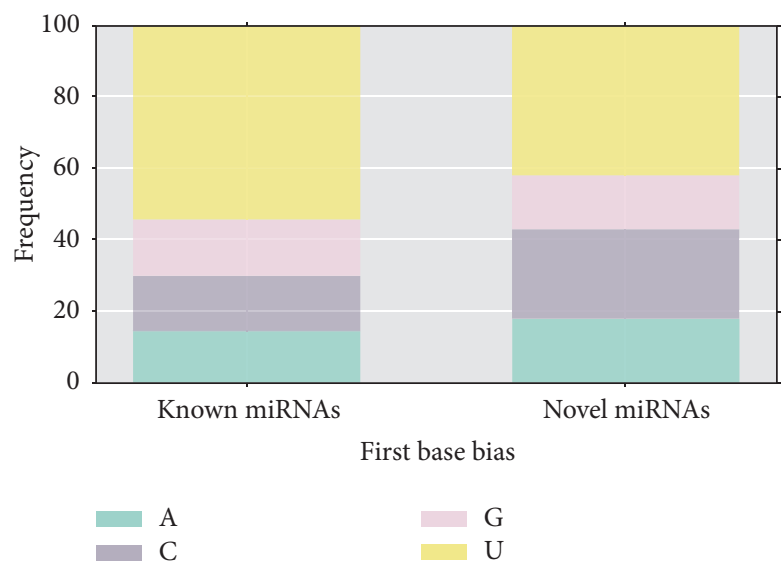

FIGURE 8: miRNA first nucleotide bias.

3.5. Target Gene Prediction for Differentially Expressed MicroRNA in "Dangshan Su" Different Pollination. According to the known interactions between miRNAs and target genes, it was found that 2-8 nucleotides in the miRNA $5^{\prime}$ end were completely complementary with UTR region in target mRNA $3^{\prime}$ end $[38,39]$. This feature is verified by a variety of target gene prediction methods. However, there are other restrictions in miRNA target gene prediction: the specific matching circumstances of miRNA $3^{\prime}$ end and the target gene, miRNA secondary structure, thermodynamic stability of the dimer of miRNA and miRNA target genes, and so on. In order to reduce target gene prediction false positive results, we used the software in plant microRNA target gene forecast, TAPIR and TargetFinder, which both have good predictions for the target genes [29]. The combined use of these two software programs leads to a number of predicted miRNA target genes and their corresponding target genes.

Among 68 differentially expressed microRNAs, 641 target genes were obtained and classified into different gene families which were involved in transcriptional regulation of stress response, signal transduction, growth, and transmembrane transport in plants (Supplementary Material_5). Regulatory network from the differentially expressed microRNA target genes and their corresponding mapping (Figure 12), was divided into six parts (Supplementary Material_6). As showed in the network, a microRNA gene can regulate multiple targets at the same time, and a target gene can also be regulated by more microRNAs. For example, pyr-miR401 and pyr-novel-miR-127-3p regulate 40 and 33 target genes, respectively, indicating that they may play an important regulatory role in pear fruit xenia phenomena. And the target gene Pbr016259.1 was regulated by 16 differentially expressed 


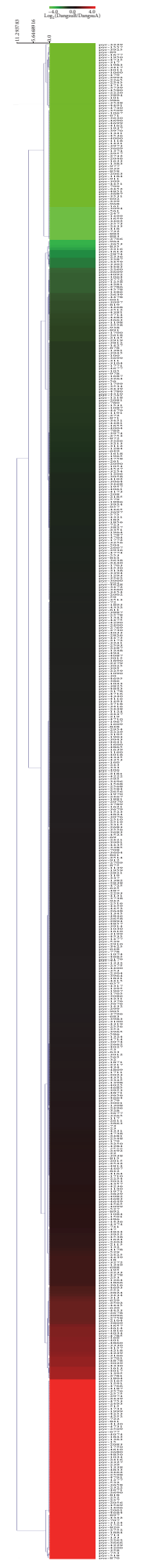

(a)

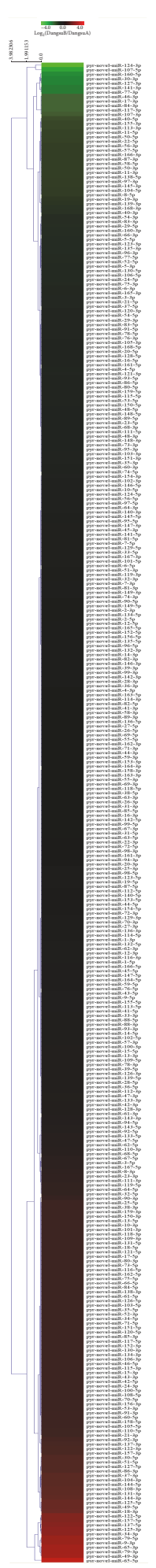

(b)

FIGURE 9: Cluster analysis of microRNA expression. (a) Expression pattern of known microRNA. (b) Expression pattern of novel microRNA. 


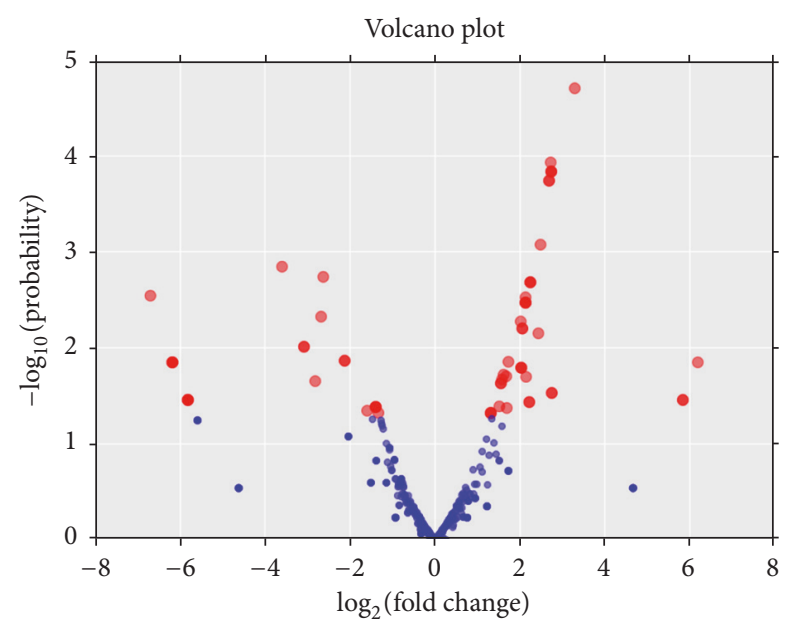

FIGURE 10: The volcano plot for differentially expressed miRNA. Note: abscissa represents the microRNA expression multiple changes in the different samples, while ordinate represents the significant level of microRNA expression changes, and blue dots indicate no significant differences in the microRNA and red dot indicates there are significant differences in microRNA.

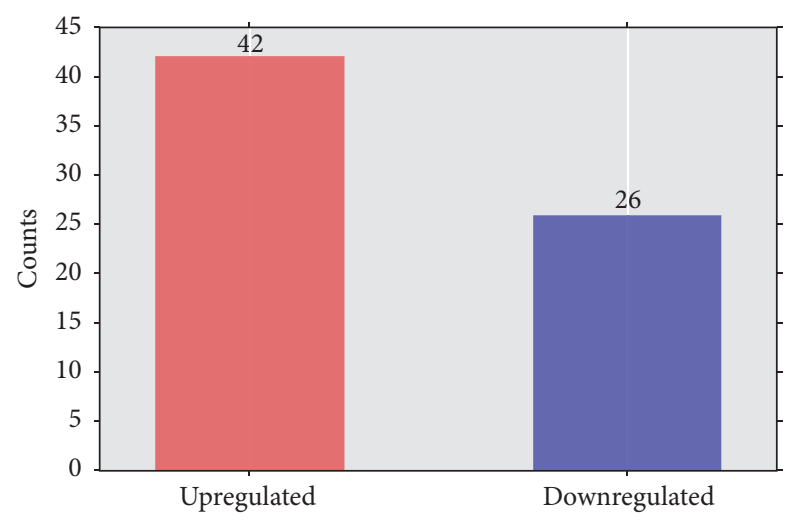

Figure 11: The number of different expressed microRNAs.

microRNAs. As Pbr016259.1 encodes for an acidic amino acid ligase, it can be inferred that acidic amino acid ligase plays an important regulatory role in pear fruit growth start, and its expression could be simultaneously controlled by multiple microRNAs in synergy regulation.

\subsection{Function Annotation of Differentially Expressed MicroRNA Target Gene in "Dangshan Su" Different Pollination}

3.6.1. GO Function Annotation of Differentially Expressed MicroRNA Target Gene. Gene Ontology (GO) is an international standardized classification system for gene function, which supplies a set of controlled vocabularies to comprehensively describe the property of genes and gene products. There are three ontologies in GO: molecular function, cellular component, and biological process. The basic unit of GO is GO term, each of which belongs to one type of ontology.
GO enrichment analysis can predict target gene candidates of novel miRNAs, as well as their biological function. The result could reveal the functions significantly related to predicted target gene candidates of novel miRNAs. As shown in Figure 13, among the differentially expressed microRNAs target genes, there are two significantly enriched functions, cellular physiological processes and metabolic processes, accounting for $45.63 \%$ and $38.75 \%$, respectively, in the entire biological process. Nucleus and film composition of cellular component were also significantly enriched, accounting for $33.33 \%$ and $46.67 \%$ of cellular component, respectively. Catalytic activity and binding combination of molecular function were significantly enriched, accounting for $29.41 \%$ and $56.30 \%$ of molecular function, severally. Moreover, most of the GO terms ( $q$ value $\leq 0.05$ ) were related to molecular function (80\%), a small amount with biological process (20\%), and no cellular component was detected (Supplementary Material_7). That means in the different pollination of "Dangshan Su," the effect of male parent to "Dangshan Su" stone cell formation and quality was altered by the microRNA expression quantity of target genes which has the molecular regulation functions and biological processes and is not related to "Dangshan Su" cellular components.

3.6.2. Pathway Analysis of Differentially Expressed MicroRNA Target Genes. KEGG pathway analysis is also used for the target gene candidates. In organisms, genes usually interact with each other to play different roles in certain biological function. The analysis based on pathways could facilitate the understanding of biological functions of genes. KEGG is the major public pathway-related database [40]. KEGG pathway analysis identifies significantly enriched metabolic pathways or signal transduction pathways in target gene candidates comparing with the whole reference gene background.

KEGG pathway analysis of the differentially expressed microRNA target genes (Supplementary Material_8) suggested that 68 target genes were enriched into 52 KEGG pathways. When the parameter $p$ value $\leq 0.05$, the significant enrichment of KEGG pathways mainly has plant pathogen interactions (Ko04626), organic selenium compound metabolized (Ko00450), sulfur metabolism (Ko00920), single stick biosynthesis (Ko00902), other polysaccharides degradation (Ko00511), purine metabolism (Ko00230), and so on.

\subsection{Impact of the Differential Expressed MicroRNAs on the Lignin Metabolism in Different Pollinated "Dangshan Su"}

3.7.1. MicroRNA Target Gene Prediction for Lignin Metabolism Regulation. Stone cells are formed by secondary thickening and lignin deposition of parenchyma cell wall [32, 41]. Therefore, lignin biosynthesis, transport, and deposition are closely related to stone cell development. One feature of pear fruits is of the enrichment of stone cells during their development, which directly affects pear fruit quality. The metabolic regulation of lignin was significantly affected by microRNA. Wu et al. detected the presence of significant expression differences of miR3711, miR419, and miR5260 in different developmental stages of "Dangshan Su" and found that their common target gene was HCT which is encoding a key 


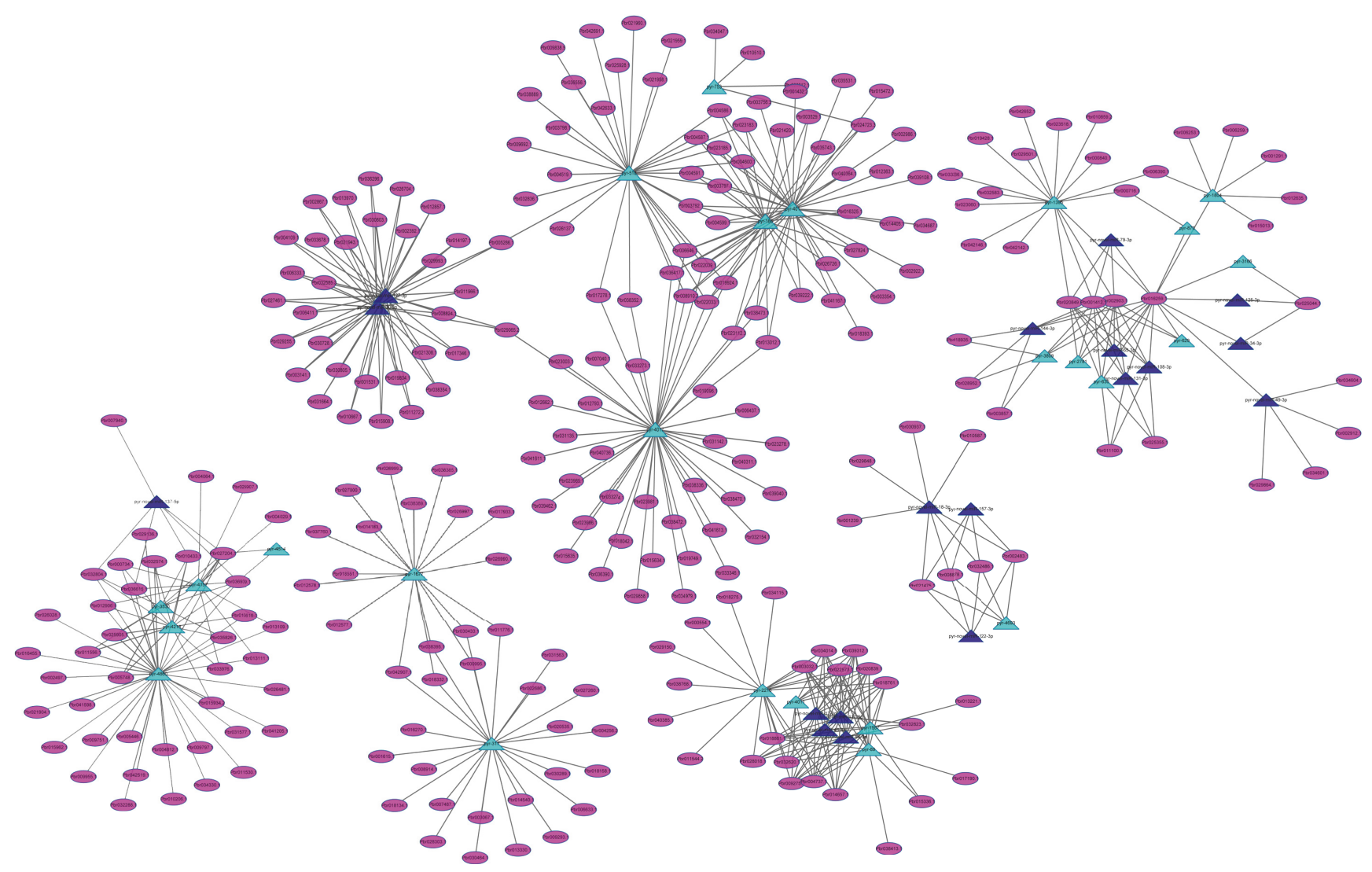

FIGURE 12: Relationship of different expression miRNAs and their target genes.

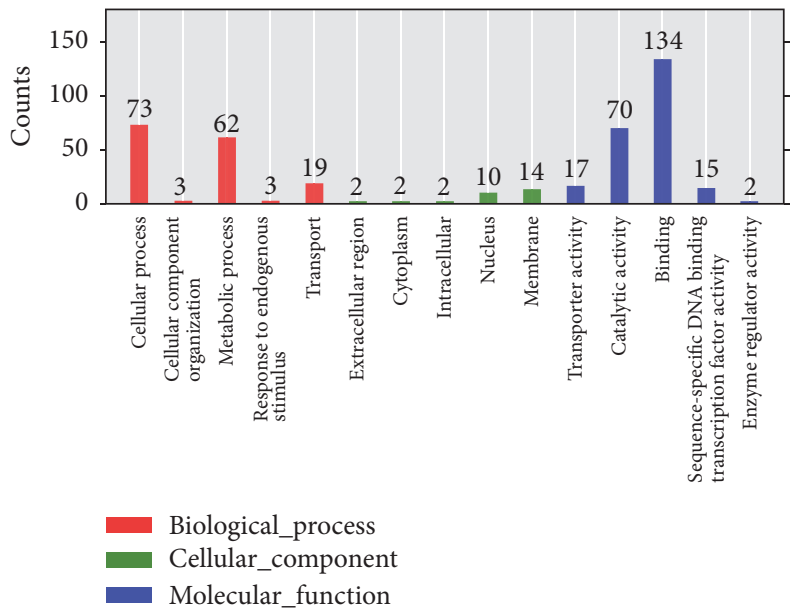

Figure 13: GO annotation class of different expressed genes.

enzyme of lignin metabolism [21]. Rubinelli et al. confirmed that overexpression of miRNA of miR156 family in transgenic Populus tomentosa can have effective influence on the tree structure and the lignin content [42]. Lu et al. proved that the ptr-miR397a overexpression significantly and negatively regulated laccase in lignin metabolic pathway and also significantly affected lignin content in Populus tomentosa [17].
Through the differentially expressed microRNA GO enrichment and KEGG pathway analysis, we discovered that the expression level of pyr-novel-miR-144-3p in DangsuB was significantly higher than that in DangsuA. Through target genes prediction of this microRNA, 6 target genes (Pbr028952.1, Pbr002903.1, Pbr018935.1, Pbr001412.1, Pbr016259.1, and Pbr003857.1) were obtained. Pbr028952.1 encodes for an ATP enzyme. Both Pbr002903.1 and Pbr001412.1 play the function of transport substrates via transmembrane. Pbr016259.1 encodes for an acidic amino acid ligase. Pbr018935.1, as well as Pbr003857.1, encodes for a laccase (PbrLAC35 or PbrLAC57, resp.) which is involved in the last key step of lignin metabolic pathways. Therefore, we proposed that via different pollination on "Dangshan Su," the different males could affect pyr-novel-miR-144$3 p$ expression, which subsequently regulated the laccase expression within lignin monomer polymerization process and thus controlled lignin and stone cells synthesis in pear fruit; this result is consistent with $\mathrm{Wu}$ and Niu [21, 23].

3.7.2. Evolutionary Analysis of Target Genes of Lignin Metabolic Regulation. To further study the homologous evolutionary relationships and functions between PbrLAC35 and PbrLAC57 in "Dangshan Su," we used NCBI BLAST to retrieve the genes which has $75 \%$ more similarity with PbrLAC35 and PbrLAC57 amino acid sequence in other species. The online 
TABLE 3: Protein properties in different species which are similar to PbrLAC35 protein sequences.

\begin{tabular}{|c|c|c|c|c|c|}
\hline Gene name & Accession number & Length (aa) & Molecular weight (Da) & $\mathrm{pI}$ & $\begin{array}{c}\text { Subcellular } \\
\text { localization } \\
\text { prediction }\end{array}$ \\
\hline MonoL484 & EXC10697.1 & 577 & 63851.3 & 9.61 & Chlo \\
\hline PhdaLAC10 & XP_008778578.1 & 577 & 63139.5 & 9.23 & Chlo \\
\hline CiarLAC17 & XP_004492322.1 & 580 & 64299.7 & 8.91 & Chlo \\
\hline CisiLAC17 & XP_006480873.1 & 578 & 63944.6 & 9.44 & Chlo \\
\hline CumeLAC17 & XP_008463808.1 & 578 & 64278.1 & 9.46 & Chlo \\
\hline LituLAC & AAB17194.1 & 585 & 64720.1 & 9.54 & Chlo \\
\hline EugrLAC17 & XP_010031544.1 & 585 & 64702.3 & 8.92 & Vacu \\
\hline Cocal & CDP09087.1 & 587 & 64180.4 & 8.94 & Chlo \\
\hline FrveLAC17 & XP_004294637.1 & 584 & 64356.1 & 9.33 & Chlo \\
\hline GsoLAC17 & KHN32142.1 & 590 & 65611 & 9.02 & Chlo \\
\hline GmaLAC17 & XP_006602563.1 & 578 & 63733.2 & 9.24 & Chlo \\
\hline Jacu05 & KDP44151.1 & 576 & 63764.9 & 9.3 & E.R. \\
\hline$M d m L A C 2$ & XP_008356891.1 & 584 & 64570.4 & 9.68 & Chlo \\
\hline MirLAC1 & КEH22816.1 & 580 & 64124.6 & 9.35 & Chlo \\
\hline NenuLAC17 & XP_010256874.1 & 583 & 64130.5 & 8.88 & Chlo \\
\hline NisyLAC17 & XP_009774063.1 & 581 & 64525.3 & 9.05 & Chlo \\
\hline NitoLAC17 & XP_009587566.1 & 581 & 64628.3 & 8.97 & Chlo \\
\hline Phvu008 & ESW12292.1 & 625 & 68966 & 9.13 & Chlo \\
\hline Ptc0001 & EEE83028.2 & 576 & 63551.8 & 9.27 & Chlo \\
\hline Рpe003 & EMJ09215.1 & 587 & 64588.1 & 9.77 & Chlo \\
\hline TccLAC17 & EOY13993.1 & 577 & 63443.8 & 9.16 & Chlo \\
\hline VviLAC17 & XP_002284473.1 & 585 & 64295.7 & 8.71 & E.R. \\
\hline RicoLAC1 & XP_002531565.1 & 577 & 63727.7 & 9.47 & Chlo \\
\hline BrraLAC17 & XP_009120521.1 & 573 & 63648.2 & 9.33 & E.R. \\
\hline MuacLAC4 & XP_009403441.1 & 579 & 63250.4 & 8.97 & Vacu \\
\hline CusaLAC17 & XP_004148786.1 & 579 & 64451.3 & 9.42 & Chlo \\
\hline PpeLAC17 & XP_008222577.1 & 581 & 64247 & 9.19 & Chlo \\
\hline PbrLAC35 & Pbr018935.1 & 585 & 64538.4 & 9.79 & Chlo \\
\hline
\end{tabular}

analysis tools like ExPAsy (http://www.expasy.org/) and WoLF PSORT (http://www.genscript.com/wolf-psort.html) were used to screen essential information of amino acids, such as length, molecular weight, isoelectric point, and subcellular localization of proteins (Tables 3 and 4). Proteins similar to PbrLAC35 and PbrLAC57 sequence in various species have the molecular weight between $63 \mathrm{kD}$ and $68 \mathrm{kD}$ and isoelectric point greater than 8.71, belonging to basic protein. And most of them are localized in chloroplasts and vacuole.

In order to further analyze homologous evolutionary relationships of PbrLAC35 and PbrLAC57 in different species, we used ClustalW tool in MEGA6.0 software to run the gene multiple sequence alignment for the amino acid sequences in different species then used neighbor-joining method (NJ) (bootstrap $=1000)$ to build composite evolutionary trees of different species. As showed in Figure 14(a), similar sequences from 27 species can be divided into three categories (I, II, and
III). The predicted target gene PbrLAC35 was targeted in class I and united with the apple MdmLAC2, and then the yellow peach Ppe003 was grouped into a branch with bootstrap support rate up to $99 \%$. And this branch subsequently combined with the GsoLAC17 of the soybean, the MonoL484 of the morus, the Ptc0001 of western balsam poplar, and the Cocal of coffee to form class I. As shown in another phylogenic tree (Figure 14(b)), which was constructed with similar sequences from 28 species and composed of two classes (I and II), the predicted target gene PbrLAC57 was located in class I. Similar to the clustering of PbrLAC35, PbrLAC57 was firstly combined with MdmLAC2; then peach PpeLAC2 was clustered into a branch supported by bootstrap up to $100 \%$. But it can also be seen that the bootstrap support rate of the later clustering is relatively low, only $50 \%$ and $82 \%$, respectively, which also shows that the conservatism of the target gene prediction in the close genetic relationship 
TABLE 4: Protein properties in different species which are similar to PbrLAC57 protein sequences.

\begin{tabular}{|c|c|c|c|c|c|}
\hline Gene name & Accession number & Length (aa) & Molecular weight $(\mathrm{Da})$ & $\mathrm{pI}$ & $\begin{array}{c}\text { Subcellular } \\
\text { localization } \\
\text { prediction }\end{array}$ \\
\hline GmaLAC2 & XP_006592190.1 & 588 & 65380.7 & 9.10 & Chlo \\
\hline$M d m L A C 2$ & XP_008384938.1 & 585 & 64520.8 & 9.63 & Chlo \\
\hline NenuLAC17 & XP_010264408.1 & 591 & 65467.7 & 9.17 & Chlo \\
\hline GsoLAC17 & KHN16226.1 & 578 & 63795.2 & 9.19 & E.R. \\
\hline CiclLAC17 & XP_006480873.1 & 578 & 63944.6 & 9.44 & Chlo \\
\hline NisyLAC2 & XP_009796024.1 & 583 & 64518.8 & 9.14 & Chlo \\
\hline RicoLAC1 & EEF30831.1 & 577 & 63727.9 & 9.47 & Chlo \\
\hline CiarLAC17 & XP_004492318.1 & 580 & 64073.6 & 9.35 & Chlo \\
\hline Ptc0001 & EEE83028.2 & 576 & 63551.8 & 9.27 & Chlo \\
\hline CumLAC17 & XP_008463808.1 & 578 & 64278.1 & 9.46 & Chlo \\
\hline MuacLAC4 & XP_009403441.1 & 579 & 63250.4 & 8.97 & Vacu \\
\hline NisyLAC17 & XP_009796361.1 & 581 & 64514.1 & 9.05 & Chlo \\
\hline Phvu011 & ESW04043.1 & 607 & 67189.9 & 9.41 & Chlo \\
\hline Jacu05 & KDP44151.1 & 576 & 63764.9 & 9.30 & E.R. \\
\hline EugrLAC17 & XP_010031544.1 & 585 & 64702.3 & 8.92 & Vacu \\
\hline VviLAC17 & XP_003632204.1 & 585 & 64388.2 & 9.02 & Chlo \\
\hline CisiLAC17 & XP_006488364.1 & 583 & 64515.8 & 9.25 & Chlo \\
\hline GsoLAC2 & XP_006592189.1 & 590 & 65611.0 & 9.02 & Chlo \\
\hline Cocal & CDP09087.1 & 587 & 64180.4 & 8.94 & Chlo \\
\hline CusaLAC17 & XP_004148786.1 & 579 & 64451.3 & 9.42 & Chlo \\
\hline MonoL484 & XP_010092993.1 & 586 & 64520.2 & 9.28 & Chlo \\
\hline MirLAC1 & XP_003623041.1 & 581 & 64658.1 & 9.16 & Vacu \\
\hline TccLAC17 & XP_007016584.1 & 577 & 63460.1 & 9.20 & Chlo \\
\hline BevuLAC17 & XP_010690924.1 & 582 & 64862.8 & 9.33 & Chlo \\
\hline StuLAC17 & XP_004246425.1 & 571 & 63525.7 & 9.32 & Chlo \\
\hline NitoLAC17 & XP_009630096.1 & 601 & 66685.2 & 9.20 & Cyto \\
\hline Phvu008 & XP_007140298.1 & 625 & 68966.0 & 9.13 & Chlo \\
\hline Caruv1 & XP_006293883.1 & 593 & 66097.0 & 9.47 & Cyto \\
\hline PpeLAC2 & XP_008222248.1 & 587 & 64484.0 & 9.71 & Chlo \\
\hline FrveLAC17 & XP_004294637.1 & 584 & 64356.1 & 9.33 & Chlo \\
\hline TccLAC2 & XP_007016364.1 & 585 & 64146.6 & 9.39 & Chlo \\
\hline StuLAC2 & XP_006350913.1 & 584 & 64771.4 & 9.22 & Chlo \\
\hline PbrLAC57 & Pbr003857.1 & 584 & 64544.1 & 9.68 & Chlo \\
\hline
\end{tabular}

species among the species is very high, but the conservatism in different species is very low. This may be related to the two laccases function, and the major role in the Rosaceae is involved in the lignin metabolism, while in some wood and Gramineae plants, it may have participation into the lignin metabolism pathway of tree type building.

3.7.3. Differentially Expressed MicroRNAs and Their Target Genes $q R T-P C R$ Validation. In order to verify the above results, we used qRT-PCR to determine the expression patterns of both pyr-novel-miR-144-3p and its target genes $\mathrm{Pbr}$ LAC35 and PbrLAC57 at the different developmental stages of "Dangshan Su" with different pollination. It can be found that the expression of pyr-novel-miR-144-3p in DangsuA at different times after the flowering was significantly higher than that in DangsuB (Figure 15). In DangsuA, pyr-novelmiR-144-3p showed an expression trend of initial increase, subsequent decrease, and the maximum at $31 \mathrm{~d}$. Although its 


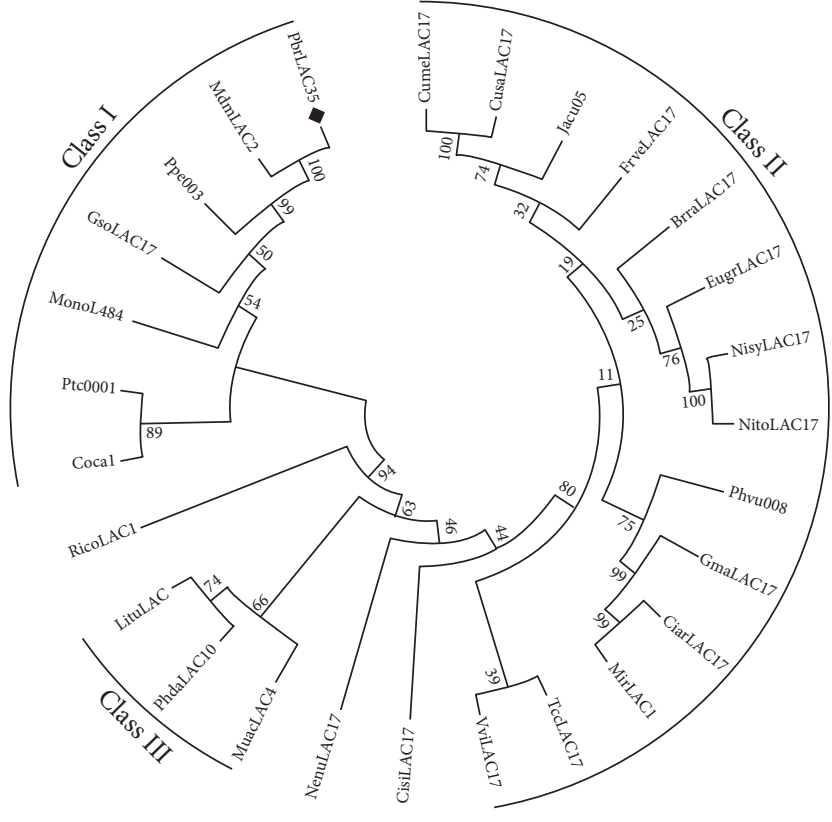

(a)

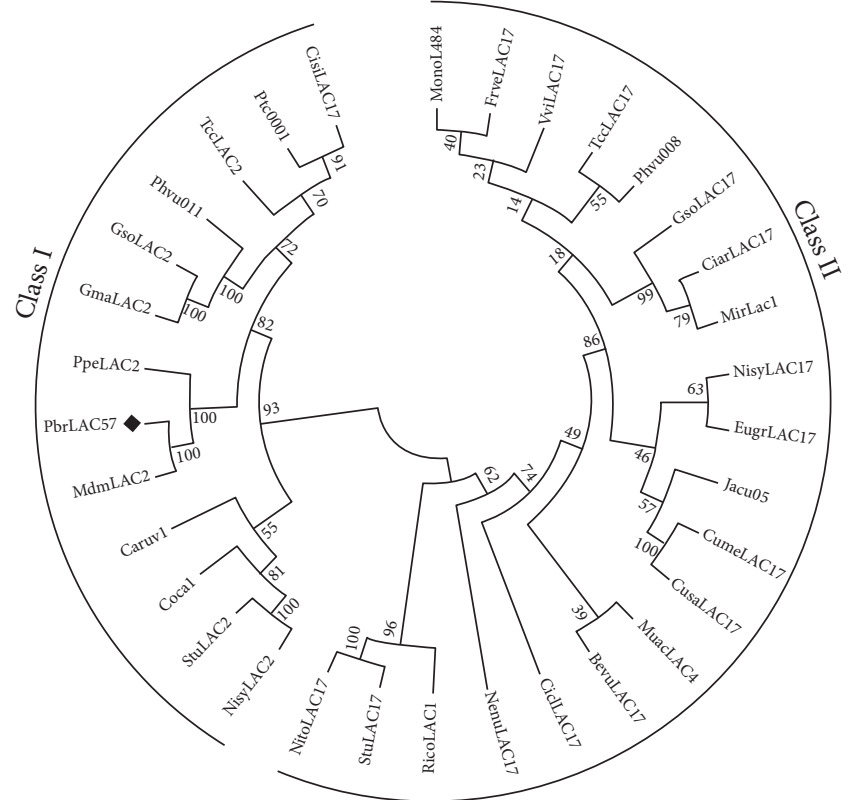

(b)

Figure 14: Phylogenetic tree of PbrLAC35 and PbrLAC57 in P. bretschneideri Rehd. constructed with neighbor-joining methods. (a) Phylogenetic tree of PbrLAC35. (b) Phylogenetic tree of PbrLAC35.

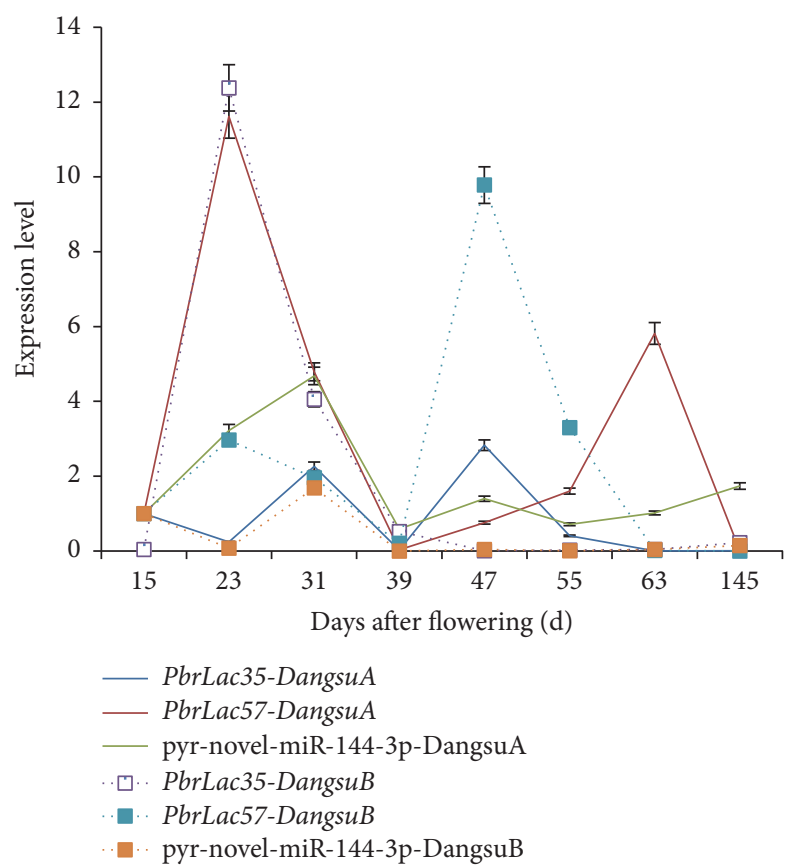

FIGURE 15: Expression pattern of miRNAs and target genes.

expression also reached a maximum at $31 \mathrm{~d}$ in DangsuB, the trend was dropping, increasing, and dropping till a stable level of expression. These results suggested that different pollination types had a significant difference in pyr-novelmiR-144-3p expression pattern. However, its expression peak at $31 \mathrm{~d}$ was similar within both pollinated "Dangshan Su," thereafter entering a stable expression period in the late stage.

In DangsuA, the target gene PbrLAC35 expression trend is down-up-down-up-down and reaches a maximum at $47 \mathrm{~d}$; PbrLAC57 expression trend is firstly up-down-up-down and reaches maximum at $23 \mathrm{~d}$. We can see that when the pyrnovel-miR-144-3p reached a maximum expression at $31 \mathrm{~d}$, PbrLAC57 expression decreased sharply from 58.08 to 4.79 and PbrLAC35 expression and the pyr-novel -miR-144-3p expression are in trade-off and taking-turns status.

In DangsuB, the expression trends of both PbrLAC35 and PbrLAC57 showed a status of fluctuation until reaching a peak at $31 \mathrm{~d}$ and $47 \mathrm{~d}$, respectively. It is noticeable that pyr-novel-miR-144-3p expression experienced a level from high to low during 15 to 23 days after flowering, while the expression levels of both PbrLAC35 and PbrLAC57 were maintained in a rising patterns. Furthermore, when pyrnovel-miR-144-3p expression began to rise from $23 \mathrm{~d}$ to $31 \mathrm{~d}$, the expression of its two target genes declined significantly. Therefore, we concluded that, in the different pollination "Dangshan Su," pyr-novel-miR-144-3p significantly regulates expression levels of laccases (PbrLAC35 and PbrLAC57) in lignin metabolic pathways. The results were consistent with previous conclusion from the target gene prediction.

According to previous reports, ptr-miR397a can significantly lower the expression of laccase in lignin synthesis regulation in Populus tomentosa [17, 21]. qRT-PCR analysis was used to verify the expression of microRNA pyr-miR1890 whose sequence is the same with ptr-miR397a. Figure 16 showed that the expression trends of pyr-miR1890 in DangsuA and DangsuB were both decreased after the first rise and 


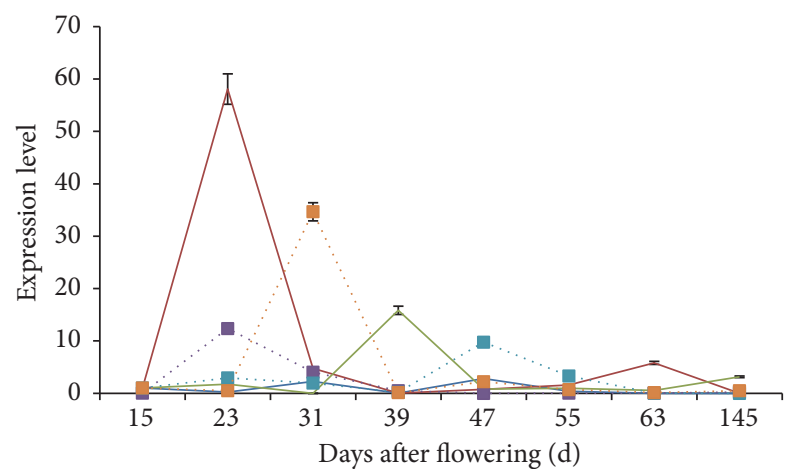

$$
\begin{aligned}
& \text { _ PbrLac35-DangsuA } \\
& \text { _ PbrLac57-DangsuA } \\
& \text { _ pyr-miR1890-DangsuA } \\
& \text { - . PbrLac35-DangsuB } \\
& \text { - . PbrLac57-DangsuB } \\
& \text {. . pyr-miR1890-DangsuB }
\end{aligned}
$$

FIGURE 16: Expression pattern of miRNAs and target genes.

reached the maximum at $39 \mathrm{~d}$ in DangsuA and at $31 \mathrm{~d}$ in DangsuB, respectively. With the increase of pyr-miR1890 expression, the expression of the two target laccases showed a significant decrease, indicating that pyr-miR1890 also played a role in the regulation of laccase (PbrLAC57 and PbrLAC35), which was consistent with the previous reports that a microRNA could regulate multiple target genes and the same target gene is regulated by multiple microRNA [43].

\subsubsection{Analysis of Lignin and Stone Cell Content in "Dangshan} Su" Different Pollination. Figure 17 showed that the trends of lignin content were firstly increasing until up to the maximum at 63 days after flowing then decreased. At 23, 31, 39, and 145 days after flowering, DangsuA lignin content was higher than DangsuB, while in other times DangsuA lignin content was less than DangsuB. The expression of PbrLAC57 was maximal at $23 \mathrm{~d}$ in DangsuA and at $47 \mathrm{~d}$ in DangsuB. Therefore, we inferred that due to the impact of xenia phenomenon, PbrLAC57 expression trend was variable in both DangsuA and DangsuB and is expressed in the early and late fruit development stage of DangsuA, while it is expressed in the mid stage of DangsuB. Moreover the DangsuA lignin content was higher than DangsuB at the development of early and late stage, while DangsuA lignin content was less than in DangsuB at the mid-stage. Thus, laccase PbrLAC57 may be a key regulatory enzyme for catalyzing lignin monomer coupling polymerization into lignin polymer.

"Dangshan Su" pear by different pollination had the same changing trend of stone cell content, increasing at first, reaching a small peak at $47 \mathrm{~d}$, and then down-up again to reach maximum at $63 \mathrm{~d}$, then decreasing. At $47 \mathrm{~d}$ and $63 \mathrm{~d}$, DangsuA stone cells content was less than DangsuB, while in other times, they were higher than DangsuB. Thus it can be seen that stone cell contents were significantly related to lignin contents with a correlation coefficient of 0.871 and 0.981 in DangsuA and DangsuB, respectively.

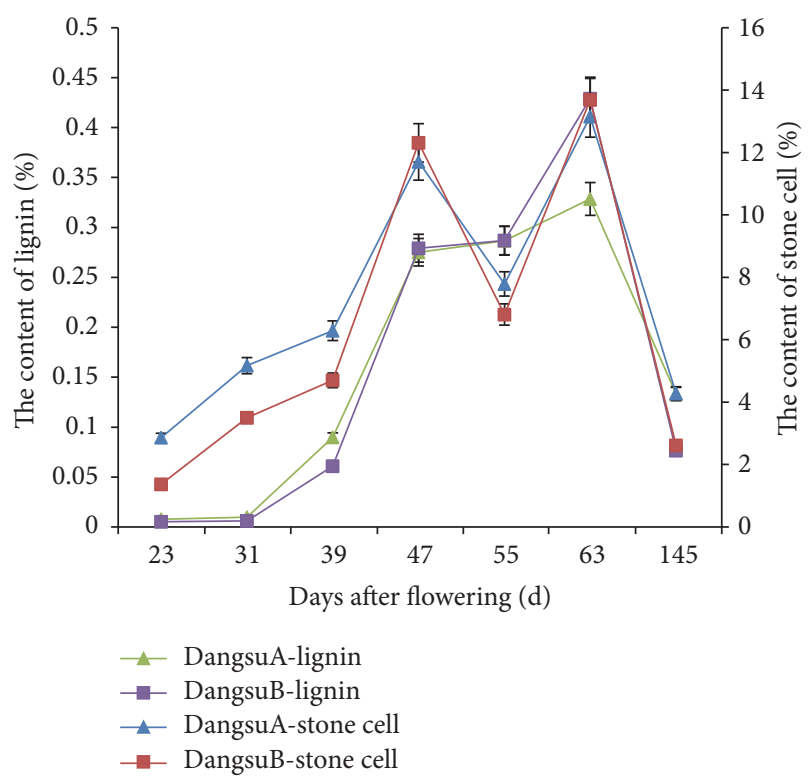

FIGURE 17: Content of stone cells and lignin in different pollination varieties of "Dangshan Su."

3.7.5. Pathway Analysis of MicroRNA Regulation in Pear Fruit Quality. According to the previous results in this study, we hypothesized that different pollination types could regulate "Dangshan Su" fruit quality through the following steps (Figure 18): (1) different males affect the expression quantity of pyr-novel-miR-144-3p and pyr-miR1890 by xenia phenomenon; (2) the expression changes of pyr-novel-miR144-3p and pyr-miR1890 result in the corresponding regulation of target gene (Pbr003857.1, Pbr028952.1, Pbr001412.1, Pbr016259.1, Pbr002903.1, and Pbr018935.1) expression level changes, especially laccases PbrLAC35 and PbrLAC57 expressions have significant difference in different developmental stages of different pollination; (3) PbrLAC35 and PbrLAC57, through expression changing, cause catalyzing of lignin monomer coupling polymerization reaction change and lignin content change; and (4) the change of lignin content results in the content change of stone cells formed by lignin deposition and achieves the effect of pear fruit quality by changing the stone cell contents.

At the same time, xenia cannot regulate lignin biosynthesis through direct effects on lignin key enzyme genes in the phenylpropanoid metabolism of the metabolic pathway upstream. Instead, it can be indirect regulation through microRNAs, which are regulators of the related laccases, to affect the synthesis of lignin and the formation of stone cells in order to control the quality of pear. This is also consistent with the principle of the evolution of species. In the critical period of lignin synthesis, the regulation can precisely catalyze the laccases for lignin monomer polymerization, which not only can accurately promote or inhibit the synthesis of lignin, but also can avoid the regulation of the upstream related gene expression and the impact of the entire secondary metabolic pathways. 


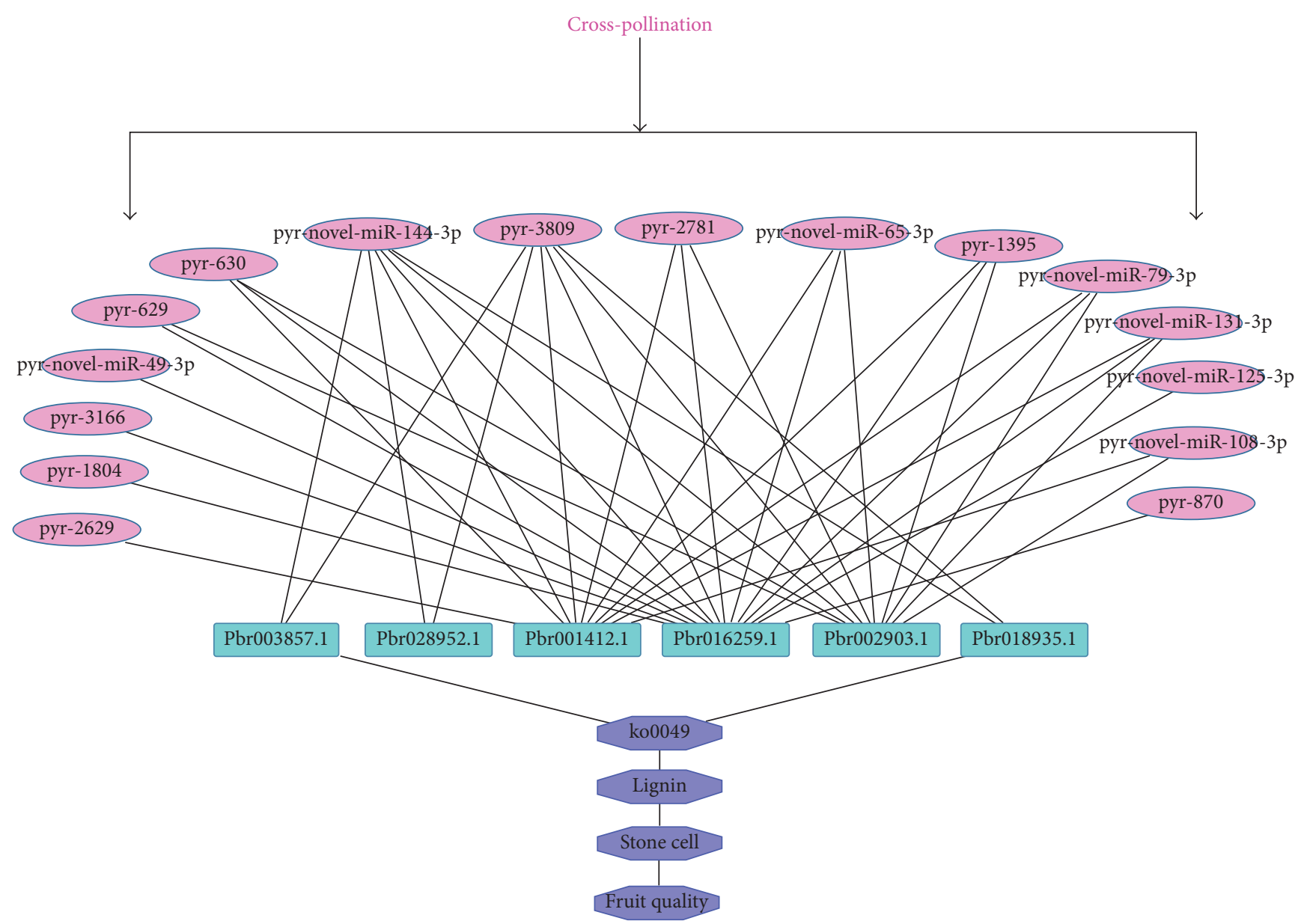

FIGURE 18: Pathway analysis of microRNA regulation in pear fruit quality.

\section{Conclusions}

We found that xenia phenomenon, instead of direct regulation, indirectly controlled lignin biosynthesis via the microRNAs which target the key genes in the phenylpropanoid metabolism, such as laccase genes. Moreover, it is found the formation of stone cells was significantly related to the lignin synthesis, which could further affect pear quality.

\section{Additional Points}

Availability and Requirements. GEO Submission (GSE80747). GSM2136459 DangsuA miRNA-seq. GSM2136460 DangsuB miRNA-seq. Database homepage: http://www.ncbi.nlm.nih .gov/geo/query/acc.cgi?acc=GSE80747.

\section{Conflicts of Interest}

The authors declare that they have no conflicts of interest.

\section{Authors' Contributions}

Xi Cheng and Chongchong Yan performed the experiments and wrote the paper. Jinyun Zhang and Chenhui
Ma contributed reagents/materials/analysis tools. Xi Cheng, Chongchong Yan, and Nan Zhang analyzed the data. Shumei $\mathrm{Li}$ and Qing Jin helped process the data. Xi Cheng and Yi Lin discussed and analyzed the results. Yongping Cai conceived and designed the experiments. All authors read and approved the final manuscript. Xi Cheng and Chongchong Yan have contributed equally to this work.

\section{Acknowledgments}

This work was supported by grants from the National Natural Science Foundation of China (nos. 31171944 and 31640068), Anhui Academy of Agricultural Sciences Dean's Youth Fund (17B0306), and Anhui Academy of Agricultural Sciences "New Dr." scientific research project.

\section{References}

[1] D. P. Bartel, "MicroRNAs: genomics, biogenesis, mechanism, and function," Cell, vol. 116, no. 2, pp. 281-297, 2004.

[2] B. Zhang, X. Pan, G. P. Cobb, and T. A. Anderson, "Plant microRNA: a small regulatory molecule with big impact," Developmental Biology, vol. 289, no. 1, pp. 3-16, 2006. 
[3] D. Baulcombe, "RNA silencing in plants," Nature, vol. 431, no. 7006, pp. 356-363, 2004.

[4] N. G. Bologna and O. Voinnet, "The diversity, biogenesis, and activities of endogenous silencing small RNAs in Arabidopsis," Annual Review of Plant Biology, vol. 65, pp. 473-503, 2014.

[5] K. Rogers and X. Chen, "Biogenesis, turnover, and mode of action of plant microRNAs," The Plant Cell, vol. 25, no. 7, pp. 2383-2399, 2013.

[6] M. Xie, S. Zhang, and B. Yu, "microRNA biogenesis, degradation and activity in plants," Cellular and Molecular Life Sciences, vol. 72, no. 1, pp. 87-99, 2015.

[7] R. C. Lee, R. L. Feinbaum, and V. Ambros, "The C. elegans heterochronic gene lin- 4 encodes small RNAs with antisense complementarity to lin-14," Cell, vol. 75, no. 5, pp. 843-854, 1993.

[8] J. Wu, H. Zhu, W. Song et al., "Identification of conservative MicroRNAs in saanen dairy goat testis through deep sequencing," Reproduction in Domestic Animals, vol. 49, no. 1, pp. 32-40, 2014.

[9] P. Liang, B. Feng, X. Zhou, and X. Gao, "Identification and developmental profiling of microRNAs in diamondback moth, Plutellaxylostella (L.)," PLoS ONE, vol. 8, no. 11, Article ID e78787, 2013.

[10] G. Chuck, H. Candela, and S. Hake, "Big impacts by small RNAs in plant development," Current Opinion in Plant Biology, vol. 12, no. 1, pp. 81-86, 2009.

[11] Z. Gao, T. Shi, X. Luo, Z. Zhang, W. Zhuang, and L. Wang, "High-throughput sequencing of small RNAs and analysis of differentially expressed microRNAs associated with pistil development in Japanese apricot," BMC Genomics, vol. 13, no. 1, article 371, 14 pages, 2012.

[12] G. F. Ferreira e Silva, E. M. Silva, M. Da Silva Azevedo et al., "microRNA156-targeted SPL/SBP box transcription factors regulate tomato ovary and fruit development," The Plant Journal, vol. 78, no. 4, pp. 604-618, 2014.

[13] R. Sunkar, Y.-F. Li, and G. Jagadeeswaran, "Functions of microRNAs in plant stress responses," Trends in Plant Science, vol. 17, no. 4, pp. 196-203, 2012.

[14] B. Khraiwesh, J.-K. Zhu, and J. Zhu, "Role of miRNAs and siRNAs in biotic and abiotic stress responses of plants," Biochimica et Biophysica Acta, vol. 1819, no. 2, pp. 137-148, 2012.

[15] E. Kaja, M. W. Szcześniak, P. J. Jensen, M. J. Axtell, T. McNellis, and I. Makałowska, "Identification of apple miRNAs and their potential role in fire blight resistance," Tree Genetics \& Genomes, vol. 11, no. 1, article 812, 11 pages, 2015.

[16] V. Eldem, U. Çelikkol Akçay, E. Ozhuner, Y. Bakır, S. Uranbey, and T. Unver, "Genome-wide identification of mirnas responsive to drought in peach (Prunus persica) by high-throughput deep sequencing," PLoS ONE, vol. 7, no. 12, Article ID e50298, 2012.

[17] S. Lu, Q. Li, H. Wei et al., "Ptr-miR397a is a negative regulator of laccase genes affecting lignin content in Populus trichocarpa," Proceedings of the National Academy of Sciences of the United States of America, vol. 110, no. 26, pp. 10848-10853, 2013.

[18] Y. L. Xu, "Outline," in Dangshan Su Pear, X. Yi-Liu, Ed., China Agriculture Press, Beijing, China, 2009.

[19] Y. L. Xu, Dangshan Su Pear, China Agriculture Press, Beijing, China, 2009.

[20] Q. Jin, C. Yan, J. Qiu, N. Zhang, Y. Lin, and Y. Cai, "Structural characterization and deposition of stone cell lignin in Dangshan Su pear," Scientia Horticulturae, vol. 155, pp. 123-130, 2013.
[21] J. Wu, D. Wang, Y. Liu, L. Wang, X. Qiao, and S. Zhang, "Identification of miRNAs involved in pear fruit development and quality," BMC Genomics, vol. 15, article no. 953, 2014.

[22] J. Wu, Z. Wang, Z. Shi et al., "The genome of the pear (Pyrus bretschneideri Rehd.)," Genome Research, vol. 23, no. 2, pp. 396408, 2013.

[23] Q. Niu, M. Qian, G. Liu, F. Yang, and Y. Teng, "A genome-wide identification and characterization of mircoRNAs and their targets in 'Suli' pear (Pyrus pyrifolia white pear group)," Planta, vol. 238, no. 6, pp. 1095-1112, 2013.

[24] S. W. Burge, J. Daub, R. Eberhardt et al., "Rfam 11.0: 10 years of RNA families," Nucleic Acids Research, vol. 41, no. 1, pp. D226D232, 2012.

[25] A. Kozomara and S. Griffiths-Jones, "MiRBase: annotating high confidence microRNAs using deep sequencing data," Nucleic Acids Research, vol. 42, no. 1, pp. D68-D73, 2014.

[26] M. Wen, Y. Shen, S. Shi, and T. Tang, "MiREvo: an integrative microRNA evolutionary analysis platform for next-generation sequencing experiments," BMC Bioinformatics, vol. 13, no. 1, article 140, 10 pages, 2012.

[27] M. R. Friedländer, S. D. MacKowiak, N. Li, W. Chen, and N. Rajewsky, "MiRDeep2 accurately identifies known and hundreds of novel microRNA genes in seven animal clades," Nucleic Acids Research, vol. 40, no. 1, pp. 37-52, 2012.

[28] B. H. Zhang, X. P. Pan, S. B. Cox, G. P. Cobb, and T. A. Anderson, "Evidence that miRNAs are different from other RNAs," Cellular and Molecular Life Sciences, vol. 63, no. 2, pp. 246-254, 2006.

[29] P. K. Srivastava, T. R. Moturu, P. Pandey, I. T. Baldwin, and S. P. Pandey, "A comparison of performance of plant miRNA target prediction tools and the characterization of features for genome-wide target prediction," BMC Genomics, vol. 15, no. 1, article no. 348, 2014.

[30] R. Shi, Y. H. Sun, X. H. Zhang, and V. L. Chiang, "Poly(T) adaptor RT-PCR," in Next-Generation MicroRNA Expression Profiling Technology: Methods and Protocols, vol. 822, pp. 53-66, Humana Press, 2012.

[31] K. J. Livak and T. D. Schmittgen, "Analysis of relative gene expression data using real-time quantitative PCR and the $2^{-\triangle \Delta C T}$ method," Methods, vol. 25, no. 4, pp. 402-408, 2001.

[32] Y. Cai, G. Li, J. Nie et al., "Study of the structure and biosynthetic pathway of lignin in stone cells of pear," Scientia Horticulturae, vol. 125, no. 3, pp. 374-379, 2010.

[33] S. Raiskila, M. Pulkkinen, T. Laakso et al., "FTIR spectroscopic prediction of Klason and acid soluble lignin variation in norway spruce cutting clones," Silva Fennica, vol. 41, no. 2, pp. 351-371, 2007.

[34] R. Karlova, J. C. Van Haarst, C. Maliepaard et al., "Identification of microRNA targets in tomato fruit development using highthroughput sequencing and degradome analysis," Journal of Experimental Botany, vol. 64, no. 7, pp. 1863-1878, 2013.

[35] C. Song, C. Wang, C. Zhang et al., "Deep sequencing discovery of novel and conserved microRNAs in trifoliate orange (Citrus trifoliata)," BMC Genomics, vol. 11, no. 1, article no. 431, 2010.

[36] Z. S. Zhou, S. Q. Huang, and Z. M. Yang, "Bioinformatic identification and expression analysis of new microRNAs from Medicago truncatula," Biochemical and Biophysical Research Communications, vol. 374, no. 3, pp. 538-542, 2008.

[37] A. C. Mallory and H. Vaucheret, "Functions of microRNAs and related small RNAs in plants," Nature Genetics, vol. 38, pp. S31S36, 2006. 
[38] E. Allen, Z. Xie, A. M. Gustafson, and J. C. Carrington, "microRNA-directed phasing during trans-acting siRNA biogenesis in plants," Cell, vol. 121, no. 2, pp. 207-221, 2005.

[39] R. Schwab, J. F. Palatnik, M. Riester, C. Schommer, M. Schmid, and D. Weigel, "Specific effects of microRNAs on the plant transcriptome," Developmental Cell, vol. 8, no. 4, pp. 517-527, 2005.

[40] M. Kanehisa, M. Araki, S. Goto et al., "KEGG for linking genomes to life and the environment," Nucleic Acids Research, vol. 36, no. 1, pp. D480-D484, 2008.

[41] C. Yan, M. Yin, N. Zhang et al., "Stone cell distribution and lignin structure in various pear varieties," Scientia Horticulturae, vol. 174, no. 1, pp. 142-150, 2014.

[42] P. M. Rubinelli, G. Chuck, X. Li, and R. Meilan, "Constitutive expression of the Corngrass1 microRNA in poplar affects plant architecture and stem lignin content and composition," Biomass and Bioenergy, vol. 54, pp. 312-321, 2013.

[43] D. P. Bartel, "MicroRNAs: target recognition and regulatory functions," Cell, vol. 136, no. 2, pp. 215-233, 2009. 

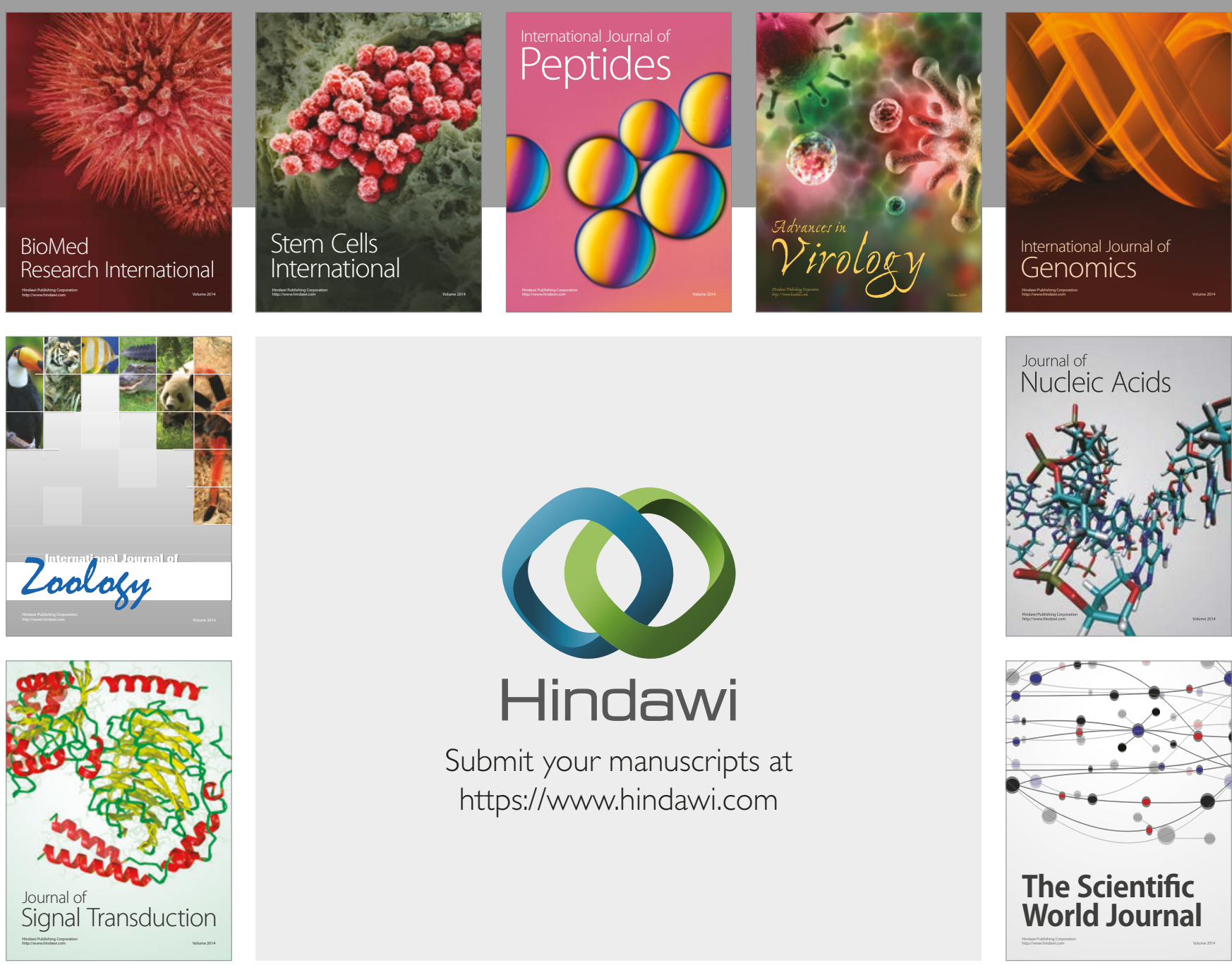

Submit your manuscripts at

https://www.hindawi.com
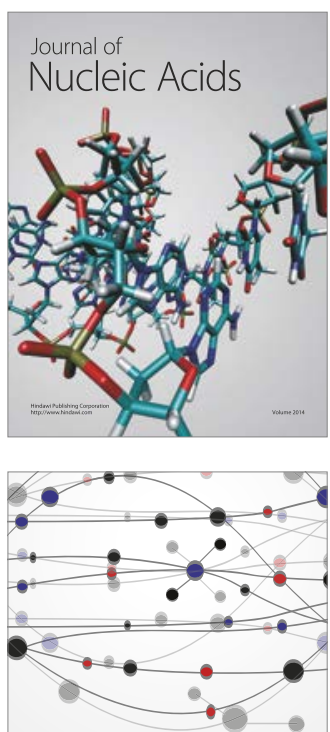

The Scientific World Journal

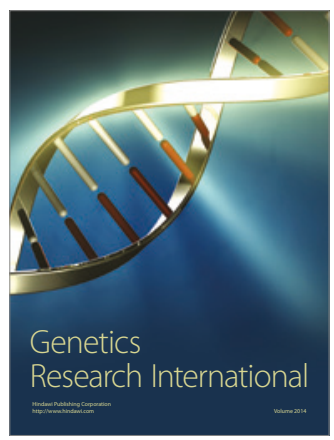

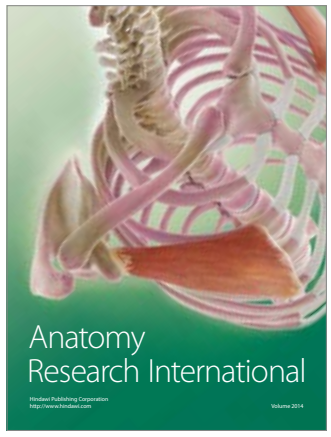

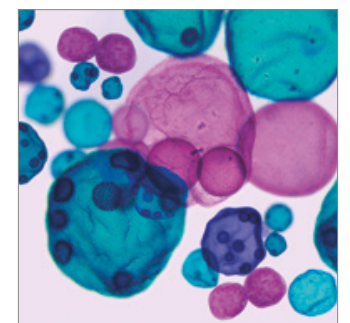

International Journal of Microbiology
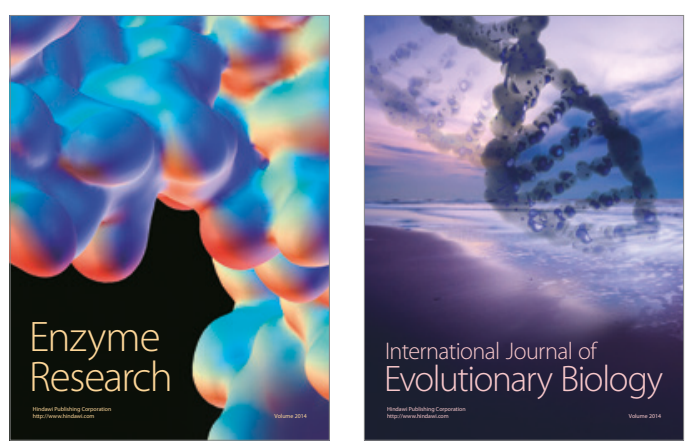
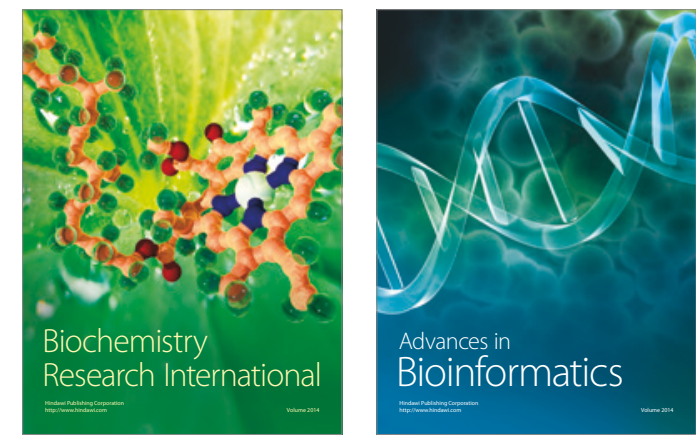

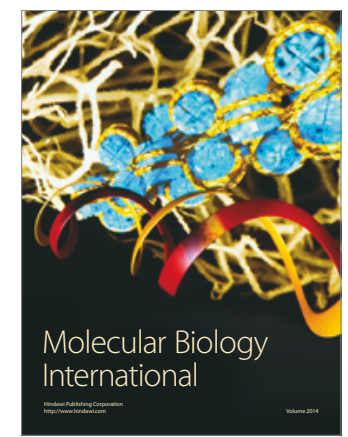

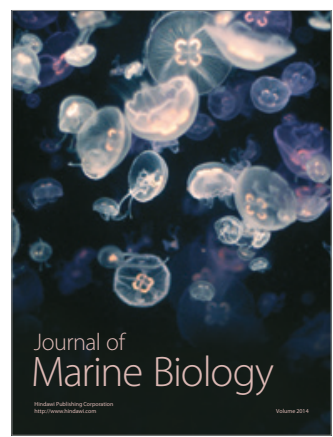

